\title{
Análisis comparativo de los planes de estudio de los programas de pregrado en filosofía en Colombia, segunda parte: contenidos ${ }^{1}$
}

\author{
Comparative Analysis of the Curricula of Undergraduate \\ Programs in Philosophy in Colombia, Part Two: Content
}

\author{
Análise comparativa das grades curriculares dos programas de \\ graduação em filosofia na Colômbia, segunda parte: conteúdos
}

René Alejandro Farieta Barrera²

\section{Resumen}

Se presenta un análisis de los planes de estudio de los 55 programas de pregrado en filosofía en Colombia. Se tipifican de acuerdo con cinco criterios: título otorgado (profesional/licenciado); especificidad disciplinar (filosofía-filosofía y otras disciplinas); tipo de registro (calificado/ alta calidad); modalidad (presencial/distancia) y sector (oficial/privado). Se presenta un modelo de clasificación de espacios académicos de los programas y lo aplicamos a todos los planes de estudio. A continuación, se presenta las medias y tendencias de acuerdo con la clasificación de los programas; primero comparando todos los programas, luego examinando por aparte los programas profesionales y posteriormente las licenciaturas. Se concluye que las principales diferencias significativas se dan entre los programas de universidades públicas y de las privadas, y también entre los programas presenciales y los que son a distancia. Por último, se muestran las implicaciones que tendrá la entrada en vigencia de una nueva normatividad para las licenciaturas en Colombia, de manera que a futuro sea posible hacer una evaluación del impacto de las nuevas políticas en la enseñanza en filosofía a nivel universitario en Colombia.

\section{Palabras clave}

filosofía; educación superior; planes de estudio; licenciatura; Colombia

\section{Abstract}

In this paper we analyze the curricula of the 55 undergraduate programs in philosophy in Colombia. We categorize them based on five criteria: title granted (professional); specific discipline (philosophy-philosophy and other disciplines); type of certification (qualified/ high quality); modality (on campus/online); and sector (official/private). We present a model to classify the academic spaces of the programs and apply it to all the curricula. Then, we present the means and trends according to the classification of the programs, first by comparing all the programs, and then by examining the professional programs and the degrees separately. We conclude that the main significant differences are between the programs from public and private universities, and also between programs taught on campus and online. Finally, we show the implications of the entry into force of a new regulation for bachelor's degrees in Colombia, so that in the future it is possible to evaluate the impact of the new policies on philosophy teaching at the university level in Colombia.

\section{Keywords}

philosophy; higher education; curricula; bachelor's degree; Colombia

1 La primera parte de este estudio se presentó en Farieta, Gómez y Almeida (2015). Este trabajo se culminó en el marco del proyecto de investigación INV-2016I-027, de convocatoria interna gestionada por la Universitaria Agustiniana. Agradezco especialmente a Marcela del Pilar Gómez por sus aportes conceptuales para refinar algunas de las categorías de espacios académicos, así como sobre algunas de las conclusiones de este estudio. También, a Sergio Almeida, Fredy Alejandro Gómez, Natalia Morales Jaramillo y Freddy Bustos por su aporte en la recopilación y sistematización de los planes de estudios.

2 Universidad Agustiniana, Bogotá, Colombia. Correo electrónico: rene.farietab@uniagustiniana.edu.co 


\section{Resumo}

Neste texto, apresenta-se uma análise das grades curriculares dos 55 programas de graduação em filosofia na Colômbia. Estes se classificaram segundo cinco critérios: título obtido (profissional/licenciado); especificidade disciplinar (filosofia/filosofia e outras disciplinas); tipo de registro (qualificado/alta qualidade); modalidade (presencial/distância) e setor (público/privado). Apresenta-se um modelo de classificação de espaços acadêmicos dos programas aplicado a todos os grades curriculares. A seguir, apresentamos as médias e tendências segundo a classificação dos programas, primeiro comparando todos os programas, depois examinando individualmente todos os programas profissionais e as licenciaturas. Como conclusão, as diferenças significativas evidenciam-se entre os programas de universidades públicas e os das privadas, também entre os programas presenciais e à distância. Finalmente, apresentam-se as implicações de uma nova normatividade para as licenciaturas na Colômbia, de forma que no futuro seja possível realizar uma avaliação do impacto das novas políticas do ensino de filosofia nas universidades na Colômbia.

\section{Palavras-chave}

filosofia; educação superior; grades curriculares; licenciatura; Colômbia

Artículo recibido el 31 de marzo de 2017 y aprobado el 10 de enero de 2018

\section{Introducción}

A continuación, presentamos un análisis de los planes de estudios de los programas de pregrado que se encontraban registrados y activos en Colombia en el primer semestre de 2016, en cuyo nombre aparece la expresión "filosofía" . De acuerdo con la información de la base de datos del Sistema Nacional de Información en Educación Superior (sNIEs), adscrita al Ministerio de Educación Nacional (MEN) de Colombia, para el 31 de mayo de ese año había cincuenta y cinco (55) programas de pregrado activos con esta característica, en treinta y seis (36) universidades; todos ellos han sido tomados para el presente análisis. Para facilitar la presentación de la información, identificaremos a cada programa con su código sNiEs.

De acuerdo con la normatividad vigente en Colombia, "el plan de estudios es el esquema estructurado de las áreas obligatorias y fundamentales y de áreas optativas con sus respectivas asignaturas que forman parte del currículo de los establecimientos educativos" (Ley 115 de 1994, art. 79). Este estudio tiene por objetivo principal examinar las

3 Este trabajo es la continuación del estudio presentado en Farieta, Gómez y Almeida (2015), cuyo objetivo general era examinar las generalidades de los programas, el número de créditos y el componente flexible de los planes de estudio. Esta segunda parte examina los componentes de los planes de estudios por tipo de espacio académico. características en la composición de los planes de estudios de estos programas académicos de pregrado, en términos del número de créditos por tipo de asignatura, para identificar las características de la educación en filosofía a nivel profesional en Colombia. Se analizan y comparan los programas de acuerdo con la siguiente tipología -la misma empleada en un estudio anterior (Farieta, Gómez y Almeida, 2015)-:

- Título otorgado: profesional o licenciado.

- Sector de la institución: oficial o privada.

- Especificidad disciplinar: filosofía o filosofía y otras (letras, humanidades, ciencias religiosas, etc.).

- Registro ante el SNIEs: r. calificado o r. de alta calidad $^{4}$.

- Modalidad: presencial o distancia (incl. virtual) ${ }^{5}$.

4 El registro calificado es la licencia que concede el MEN en Colombia cuando el programa demuestra cumplir con las condiciones de calidad exigidas para los programas de educación superior, reglamentadas por el Decreto 1295 de 2010 y 1075 de 2015 del MEN. El registro de acreditación de alta calidad es un reconocimiento del MEN a los programas que demuestran excelencia (CNA, 2013). El registro calificado es obligatorio, mientras que la acreditación de alta calidad es un proceso voluntario.

5 Aunque hay dos modalidades de programas académicos de filosofía a distancia en Colombia, de acuerdo con la información que ofrece el SNIES (distancia tradicional y virtual), hemos agrupado estas dos modalidades en un solo ítem, puesto que solamente hay un programa virtual (sNIES 9570). 
Tabla 1. Programas de pregrado activos en Colombia el primer semestre de 2016, en cuyo nombre se encuentra la expresión "filosofía"

\begin{tabular}{|c|c|c|c|c|c|c|}
\hline $\begin{array}{l}\text { Cód. } \\
\text { SNIES }\end{array}$ & Nombre de la institución & $\begin{array}{c}\text { Nombre del } \\
\text { programa }\end{array}$ & Sector & $\begin{array}{l}\text { Tipo de } \\
\text { registro }\end{array}$ & Modalidad & Municipio (dpto.) \\
\hline 20 & Universidad Nacional de Colombia & Filosofía & Oficial & Alta calidad & Presencial & Bogotá D.C \\
\hline 295 & Universidad de Caldas & Filosofía y Letras & Oficial & Alta calidad & Presencial & Manizales (Caldas) \\
\hline 417 & Universidad de Antioquia & Lic. en Filosofía & Oficial & Alta calidad & Presencial & Medellín (Antioquia) \\
\hline 441 & Universidad de Antioquia & Filosofía & Oficial & Alta calidad & Presencial & Medellín (Antioquia) \\
\hline 557 & Universidad del Valle & Lic. en Filosofía & Oficial & Alta calidad & Presencial & $\begin{array}{l}\text { Cali (Valle del } \\
\text { Cauca) }\end{array}$ \\
\hline 744 & Universidad de Cartagena & Filosofía & Oficial & Calificado & Presencial & Cartagena (Bolívar) \\
\hline 773 & Universidad Sergio Arboleda & $\begin{array}{l}\text { Lic. en Filosofía y } \\
\text { Humanidades }\end{array}$ & Privada & Alta calidad & Presencial & Bogotá D.C. \\
\hline 933 & Pontificia Universidad Javeriana & Lic. en Filosofía & Privada & Calificado & Presencial & Bogotá D.C. \\
\hline 955 & Pontificia Universidad Javeriana & Filosofía & Privada & Alta calidad & Presencial & Bogotá D.C. \\
\hline 1077 & Universidad Santo Tomás & $\begin{array}{l}\text { Lic. en Filosofía y } \\
\text { Educación Religiosa }\end{array}$ & Privada & Calificado & $\begin{array}{l}\text { Distancia } \\
\text { tradicional }\end{array}$ & Bogotá D.C. \\
\hline 1197 & Universidad Pontificia Bolivariana & Filosofía & Privada & Alta calidad & Presencial & Medellín (Antioquia) \\
\hline 1300 & $\begin{array}{l}\text { Colegio Mayor de Nuestra } \\
\text { Señora del Rosario }\end{array}$ & Filosofía & Privada & Alta calidad & Presencial & Bogotá D.C. \\
\hline 1371 & Universidad de San Buenaventura & Lic. en Filosofía & Privada & Alta calidad & Presencial & Bogotá D.C. \\
\hline 1445 & Universidad de la Salle & Filosofía y Letras & Privada & Alta calidad & Presencial & Bogotá D.C. \\
\hline 1537 & Universidad de los Andes & Filosofía & Privada & Alta calidad & Presencial & Bogotá D.C. \\
\hline 1872 & Universidad Sergio Arboleda & $\begin{array}{l}\text { Filosofía y } \\
\text { Humanidades }\end{array}$ & Privada & Alta calidad & Presencial & Bogotá D.C. \\
\hline 2049 & $\begin{array}{l}\text { Corporación Universitaria } \\
\text { Minuto de Dios }\end{array}$ & Lic. en Filosofía & Privada & Alta calidad & Presencial & Bogotá D.C. \\
\hline 2715 & Universidad de Nariño & $\begin{array}{l}\text { Lic. en Filosofía } \\
\text { y Letras }\end{array}$ & Oficial & Calificado & Presencial & Pasto (Nariño) \\
\hline 2718 & Universidad Pontificia Bolivariana & $\begin{array}{l}\text { Lic. en Filosofía } \\
\text { y Letras }\end{array}$ & Privada & Calificado & Presencial & Medellín (Antioquia) \\
\hline 4074 & $\begin{array}{l}\text { Universidad Nacional } \\
\text { Abierta y a Distancia }\end{array}$ & Filosofía & Oficial & Calificado & $\begin{array}{l}\text { Distancia } \\
\text { tradicional }\end{array}$ & Bogotá D.C. \\
\hline 4378 & $\begin{array}{l}\text { Fundación Universitaria } \\
\text { Católica Lumen Gentium }\end{array}$ & $\begin{array}{l}\text { Lic. en Filosofía y } \\
\text { Ciencias Religiosas }\end{array}$ & Privada & Calificado & Presencial & $\begin{array}{l}\text { Cali (Valle del } \\
\text { Cauca) }\end{array}$ \\
\hline 4450 & $\begin{array}{l}\text { Universidad Nacional } \\
\text { Abierta y a Distancia }\end{array}$ & Lic. en Filosofía & Oficial & Calificado & $\begin{array}{l}\text { Distancia } \\
\text { tradicional }\end{array}$ & Bogotá D.C. \\
\hline 8167 & Universidad Libre & Filosofía & Privada & Calificado & Presencial & Bogotá D.C. \\
\hline 8460 & Universidad Industrial de Santander & Filosofía & Oficial & Calificado & Presencial & $\begin{array}{l}\text { Bucaramanga } \\
\text { (Santander) }\end{array}$ \\
\hline 9363 & $\begin{array}{l}\text { Corporación Universitaria } \\
\text { Minuto de Dios }\end{array}$ & Estudios en Filosofía & Privada & Alta calidad & Presencial & Bogotá D.C. \\
\hline 9570 & $\begin{array}{l}\text { Fundación Universitaria } \\
\text { Católica del Norte }\end{array}$ & $\begin{array}{l}\text { Lic. en Filosofía y } \\
\text { Educación Religiosa }\end{array}$ & Privada & Calificado & Virtual & $\begin{array}{l}\text { Santa Rosa de } \\
\text { Osos (Antioquia) }\end{array}$ \\
\hline 9693 & Universidad del Atlántico & Filosofía & Oficial & Calificado & Presencial & $\begin{array}{l}\text { Barranquilla } \\
\text { (Atlántico) }\end{array}$ \\
\hline 10264 & Universidad Tecnológica de Pereira & Lic. en Filosofía & Oficial & Alta calidad & Presencial & Pereira (Risaralda) \\
\hline 10463 & $\begin{array}{l}\text { Universidad Pedagógica y } \\
\text { Tecnológica de Colombia }\end{array}$ & Lic. en Filosofía & Oficial & Calificado & Presencial & Tunja (Boyacá) \\
\hline 10793 & Universidad Santo Tomas & $\begin{array}{l}\text { Lic. en Filosofía, } \\
\text { Pensamiento Político } \\
\text { y Económico }\end{array}$ & Privada & Calificado & $\begin{array}{l}\text { Distancia } \\
\text { tradicional }\end{array}$ & Bogotá D.C. \\
\hline 10798 & Universidad Santo Tomas & $\begin{array}{l}\text { Lic. en Filosofía, Ética } \\
\text { y Valores Humanos }\end{array}$ & Privada & Calificado & \begin{tabular}{|l|} 
Distancia \\
tradicional
\end{tabular} & Bogotá D.C. \\
\hline
\end{tabular}


Universidad Pedagógica Nacional

Facultad de Humanidades

\begin{tabular}{|c|c|c|c|c|c|c|}
\hline $\begin{array}{l}\text { Cód. } \\
\text { SNIES }\end{array}$ & Nombre de la institución & $\begin{array}{l}\text { Nombre del } \\
\text { programa }\end{array}$ & Sector & $\begin{array}{l}\text { Tipo de } \\
\text { registro }\end{array}$ & Modalidad & Municipio (dpto.) \\
\hline 11374 & Universidad del Valle & $\begin{array}{l}\text { Profesional en } \\
\text { Filosofía }\end{array}$ & Oficial & Calificado & Presencial & $\begin{array}{l}\text { Cali (Valle del } \\
\text { Cauca) }\end{array}$ \\
\hline 11927 & Universidad de Pamplona & Filosofía & Oficial & Calificado & Presencial & $\begin{array}{l}\text { Pamplona (N. } \\
\text { Santander) }\end{array}$ \\
\hline 11929 & Fundación Universitaria San Alfonso & Filosofía - Teología & Privada & Calificado & Presencial & Bogotá D.C. \\
\hline 11967 & Universidad del Cauca & Filosofía & Oficial & Calificado & Presencial & Popayán (Cauca) \\
\hline 11971 & Fundación Universitaria Luis Amigó & Filosofía & Privada & Calificado & Presencial & Medellín (Antioquia) \\
\hline 12047 & Universidad Externado de Colombia & Filosofía & Privada & Calificado & Presencial & Bogotá D.C. \\
\hline 13217 & Universidad Santo Tomas & $\begin{array}{l}\text { Lic. en Filosofía y } \\
\text { Lengua Castellana }\end{array}$ & Privada & Alta calidad & Presencial & Bogotá D.C. \\
\hline 13218 & Universidad de Caldas & $\begin{array}{l}\text { Lic. en Filosofía } \\
\text { y Letras }\end{array}$ & Oficial & Calificado & Presencial & Manizales (Caldas) \\
\hline 14826 & Universidad del Quindío & Filosofía & Oficial & Calificado & Presencial & Armenia (Quindío) \\
\hline 20444 & Fundación Universitaria Luis Amigó & Lic. en Filosofía & Privada & Calificado & Presencial & Medellín (Antioquia) \\
\hline 20650 & Fundación Universitaria Luis Amigó & Lic. en Filosofía & Privada & Calificado & $\begin{array}{l}\text { Distancia } \\
\text { tradicional }\end{array}$ & Medellín (Antioquia) \\
\hline 53049 & Universidad El Bosque & Filosofía & Privada & Calificado & Presencial & Bogotá D.C. \\
\hline 53355 & $\begin{array}{l}\text { Fundación Universidad } \\
\text { Autónoma de Colombia }\end{array}$ & Filosofía & Privada & Calificado & Presencial & Bogotá D.C. \\
\hline 53718 & Universidad Pedagógica Nacional & Lic. en Filosofía & Oficial & Calificado & Presencial & Bogotá D.C. \\
\hline 53758 & Fundación Universitaria Luis Amigó & Filosofía & Privada & Calificado & $\begin{array}{l}\text { Distancia } \\
\text { tradicional }\end{array}$ & Medellín (Antioquia) \\
\hline 54163 & Pontificia Universidad Javeriana & Filosofía & Privada & Calificado & Presencial & $\begin{array}{l}\text { Cali (Valle del } \\
\text { Cauca) }\end{array}$ \\
\hline 54345 & Fundación Universitaria San Alfonso & Lic. en Filosofía & Privada & Calificado & Presencial & Bogotá D.C. \\
\hline 90479 & Universitaria Agustiniana & Lic. en Filosofía & Privada & Calificado & Presencial & Bogotá D.C. \\
\hline 90553 & Universidad de Antioquia & Lic. en Filosofía & Oficial & Calificado & Presencial & $\begin{array}{l}\text { Carmen de Viboral } \\
\text { (Antioquia) }\end{array}$ \\
\hline 90806 & Universidad de la Sabana & Filosofía & Privada & Calificado & Presencial & $\begin{array}{l}\text { Chía } \\
\text { (Cundinamarca) }\end{array}$ \\
\hline 91044 & Universidad La Gran Colombia & $\begin{array}{l}\text { Lic. en Filosofía } \\
\text { e Historia }\end{array}$ & Privada & Calificado & Presencial & Bogotá D.C. \\
\hline 91267 & Universidad de Antioquia & Lic. en Filosofía & Oficial & Calificado & Presencial & $\begin{array}{l}\text { Caucasia } \\
\text { (Antioquia) }\end{array}$ \\
\hline 103785 & Universidad del Norte & $\begin{array}{l}\text { Filosofía y } \\
\text { Humanidades }\end{array}$ & Privada & Calificado & Presencial & $\begin{array}{l}\text { Barranquilla } \\
\text { (Atlántico) } \\
\end{array}$ \\
\hline 105065 & Universidad del Norte & $\begin{array}{l}\text { Lic. en Filosofía y } \\
\text { Humanidades }\end{array}$ & Privada & Calificado & Presencial & $\begin{array}{l}\text { Barranquilla } \\
\text { (Atlántico) } \\
\end{array}$ \\
\hline
\end{tabular}

Fuente: elaboración propia con información tomada del Sistema Nacional de Información de la Educación Superior [SNIES] (http://snies.mineducacion.gov.co/consultasnies/programa/buscar.jsp?control=0.23816498138523357).

En Colombia, las licenciaturas son programas de pregrado en educación (Ley 30 de 1992), y se distinguen de los programas profesionales, cuyo contenido es principalmente disciplinar. De acuerdo con la clasificación del sNiEs, todos los programas profesionales en filosofía corresponden al área de conocimiento de las ciencias sociales y humanas, mientras que las licenciaturas se encuentran en ciencias de la educación. La entrada en vigencia del Decreto 2450 de 2015 y la Resolución 2041 de 2016 del MEN, que reglamentan las condiciones de calidad para los programas de Licenciatura en Colombia, necesariamente supondrán cambios importantes en los planes de estudio de los programas de Licenciatura en Filosofía, que deberán ocurrir aproximadamente en los próximos dos años, de acuerdo con la nueva reglamentación. 
El presente estudio permitirá contrastar el estado actual de los programas, previo a la nueva legislación, y el estado posterior a la implementación de esta. Esto hará posible una valoración efectiva del impacto que tendrá la entrada en vigencia de esta nueva normatividad en la enseñanza de la filosofía a nivel universitario, tanto en los programas profesionales en esta área como en las licenciaturas. Esta nueva reglamentación exige cambios en los nombres de los programas y la composición de planes de estudios, en particular en su componente de prácticas y su modalidad; por lo que en la parte final del presente estudio presentaremos cuáles serán las principales implicaciones de la nueva reglamentación para los distintos programas de Licenciatura en Filosofía. Nuestro interés no es solamente aportar significativamente al estado del arte de la enseñanza de la filosofía en Colombia, sino también, con la caracterización de los programas, hacer un aporte para la discusión de cualquier reforma curricular o de plan de estudios que se realice en los próximos años en los programas de filosofía y áreas relacionadas en las universidades colombianas.

\section{Metodología}

En este estudio proponemos una clasificación de todos los espacios académicos que componen cada uno de los 55 programas estudiados, que describimos a continuación, y posteriormente estableceremos diferencias significativas en la composición de los programas de acuerdo con su tipología.

\section{Categorías de espacios académicos}

Para poder analizar y comparar los planes de estudios de los programas de filosofía, se han determinado las siguientes categorías de componentes y áreas temáticas de los espacios académicos que servirán de eje de comparación de los distintos planes de estudios. Aunque los planes de estudios tienen estructuras considerablemente diferentes, todos los espacios académicos se pueden clasificar en alguna de las siguientes categorías:

\section{Cursos de filosofía (CF)}

En esta categoría se agrupan las asignaturas disciplinares de los planes de estudios de filosofía, que por lo general son introductorias y se imparten en los primeros semestres de las carreras de filosofía, aunque en algunos casos se pueden dictar cursos de temas más específicos, para estudiantes más avanzados. Este tipo de cursos se caracteriza principalmente por ser dictado mediante la metodología de cátedra magistral, en la cual la exposición del profesor es el centro de la clase (Morel, 2004; Rodríguez, 2011). Se remonta presumiblemente a la lectio latina (Seel, 2012), en la cual los estudiantes escuchaban sin preguntar siquiera; aunque puede considerarse a la escuela pitagórica como la pionera en esta manera de educar (Jámblico, 2003, pp. 65-66).

\section{Seminarios de fillosofía [SF]}

En esta categoría se agrupan las asignaturas disciplinares, por lo general de profundización, que se ofrecen para los estudiantes avanzados y que se realizan mediante la modalidad de seminario investigativo, seminario alemán o alguna de sus variaciones (Navarro, 1983; Ordóñez, 2005). En esta metodología hay una mayor intervención de los estudiantes: usualmente cada sesión es preparada por un estudiante quien presenta una exposición o relatoría, en la cual se plantean los principales temas $y$ asuntos para ser discutidos por todos los participantes del seminario en esta sesión. Su origen, en contraposición con los cursos de filosofía, es presumiblemente la disputatio medieval (Seel, 2012). En general este tipo de espacios académicos no tienen más de quince estudiantes, cada uno de ellos con una presentación por semana de clase.

\section{Cursos metodológicos [CM]}

Esta categoría agrupa los espacios académicos en los que se brindan herramientas básicas de la disciplina, para desarrollar habilidades de argumentación, interpretación e investigación. Se incluyen principalmente cursos de lectura y escritura, argumentación, lógica, metodología de investigación, etc. Se incluye también el curso de preseminario, 
bastante común en los programas de filosofía, dado que sirve de introducción a la metodología de investigación más utilizada en filosofía, que es la de seminario investigativo. Muchos de estos cursos no tienen unos contenidos previamente definidos, sino que buscan el desarrollo de habilidades, capacidades y competencias genéricas, como las evaluadas en las pruebas Saber Pro (Gutiérrez y Flórez, 2011; Navarro, 2013).

\section{Lenguas modernas [LM]}

Comprende el número de créditos de cada plan de estudios dedicado a la enseñanza de las lenguas modernas (inglés, francés, alemán, etc.). En algunos casos este componente no hace parte del total del plan de estudios, sino de cursos independientes pero exigidos por cada universidad como requisito de grado.

\section{Lenguas clásicas [LC]}

En algunos programas sigue siendo obligatorio el estudio de alguna lengua clásica, en particular griego o latín -o ambas- por su importancia para el estudio de la filosofía antigua y medieval; si bien todavía se considera que las lenguas clásicas son el lenguaje de la filosofía por excelencia (Escobar, 2010). En esta categoría se contabilizarán los créditos dedicados a estos espacios académicos con independencia de a qué lengua correspondan.

\section{Cursos de pedagogía [PE]}

Todas las licenciaturas y algunos programas profesionales ofrecen cursos sobre educación, pedagogía o didáctica, como herramientas para la formación de maestros en filosofía. En esta categoría se contabilizarán los créditos específicos de cada programa que están dedicados a este tipo de materias. Se incluyen cursos sobre psicología educativa, sociología de la educación, didácticas específicas para filosofía o para ciencias sociales, teorías de aprendizaje y otras asignaturas recurrentes en los planes de estudio. En general se trata de cursos puramente teóricos, aunque en algunos casos estos cursos están ligados o son correquisito de espacios académicos de práctica.

\section{Electivas libres [EL]}

Casi todos los programas tienen un componente de libre elección, cuyos espacios académicos son ofrecidos por las universidades para todos sus programas, y que por lo general permite que los estudiantes tengan un acercamiento a diferentes ámbitos interdisciplinares, culturales o deportivos que redundan en una formación integral. Se trata de un componente flexible de libre elección, distinto del componente flexible disciplinar u optativo ( $c f$. Farieta, Gómez y Almeida, 2015).

\section{Cursos disciplinares no filosóficos (DNF)}

En todos los programas que combinan filosofía con otras disciplinas -letras, humanidades, ciencias religiosas, etc.-, se ha distinguido el número de créditos dedicado a estas. Esto permitirá comparar el número de créditos que estos programas dedican a los distintos campos disciplinares. También se encuentra este tipo de espacios académicos en programas en cuyo título solamente aparece la palabra filosofía, pero en los que el enfoque o el perfil profesional es más interdisciplinar.

\section{Profundización (PR)}

Se han clasificado por aparte los créditos optativos que cada programa incluye para que los estudiantes profundicen en las áreas de su interés. En los casos en los que el programa tiene distintas líneas de profundización se trata de los mismos créditos del componente flexible disciplinar ( $c f$. Farieta, Gómez y Almeida, 2015). También hay casos en los que los estudiantes pueden optar por líneas de profundización de acuerdo con la especificidad disciplinar del programa, como en los casos de los programas que combinan filosofía con alguna otra disciplina -letras, humanidades, ciencias religiosas, etc.- y los créditos de profundización son en alguna de estas disciplinas.

\section{Tecnologías de la información y la comunicación (TIC)}

Es cada vez más común que los programas incluyan en sus planes de estudio algunos cursos dedicados al aprendizaje del manejo de herramientas 
informáticas - procesadores de texto, bases de datos, herramientas de manejo de información bibliográfica, entornos y recursos virtuales de aprendizaje, software de análisis de datos, etc. (Unesco Institute for Statistics, 2009; Schalk, 2010; Ramírez y Casillas, 2014)-. Se incluyen aquí los cursos de carácter instrumental, pero también los dedicados a la reflexión a propósito de este tipo de herramientas o a su diseño.

\section{Prácticas (PRA)}

Se cuentan en esta categoría todos los créditos que dedica el programa para el desarrollo de prácticas, ya se trate de prácticas pedagógicas docentes (obligatorias para las licenciaturas) o de prácticas profesionales, que se incluyen en algunos programas profesionales.

\section{Trabajo de grado (TG)}

En esta categoría se incluyen todos los créditos dedicados a cumplir con el requisito de trabajo de grado en cada uno de los programas, sin distinguir sus modalidades -monografía, proyecto pedagógico, sistematización de prácticas, etc.--

\section{Otros (0)}

Todos los demás créditos que no hayan sido clasificados de acuerdo con las categorías anteriores se agrupan en esta categoría, que por lo general incluye cursos obligatorios no disciplinares, las cátedras institucionales $\mathrm{u}$ otro tipo de asignaturas exigidas por las instituciones.

\section{Fases del análisis de resultados}

En "Conclusiones" mostraremos los resultados de aplicar la clasificación de espacios académicos a cada plan de estudios de acuerdo con las categorías que planteamos en "Categorías de espacios académicos". Posteriormente analizaremos los resultados en cuatro fases:

Fase 1 ("Media de créditos por categoría para todos los programas"): medias de las categorías de espacios académicos teniendo en cuenta los 55 programas.
Fase 2 ("Análisis por tipología de programas"): medias y diferencias significativas de las categorías de espacios académicos discriminados por las tipologías presentadas en la introducción.

Fase 3 ("Programas profesionales en filosofía"): medias y diferencias significativas de las categorías de espacios académicos para los programas profesionales discriminados por las demás tipologías.

Fase 4 ("Licenciaturas en filosofía"): medias y diferencias significativas de las categorías de espacios académicos para las licenciaturas discriminadas por las demás tipologías.

Hemos optado por hacer énfasis en el análisis de los programas profesionales y en las licenciaturas porque el MEN considera que pertenecen a áreas de conocimiento distintas por lo que tienen requerimientos de calidad distintos. Además, como ha sido mostrado por Farieta, Gómez y Almeida (2015), en esta tipología se encuentran las mayores diferencias en términos de duración de los programas y número de créditos.

En las fases 2, 3 y 4 se resaltarán las mayores diferencias y se intentará mostrar en cada caso a qué podrían deberse estas diferencias. Para establecer las diferencias significativas entre los programas se utilizó la prueba $U$ de Mann y Whitney (1947), también conocida como la prueba de rangos de suma de Wilcoxon (1945), que es una prueba no paramétrica que permite encontrar diferencias significativas entre dos muestras independientes. El cálculo de la prueba es el siguiente:

$$
U=n_{1} n_{2}+\frac{n_{2}\left(n_{2}+1\right)}{2}-\sum_{i=n_{2}+1}^{n_{2}} R_{1}
$$

Para la prueba $U$ hemos establecido un $\alpha=0,1$. En todos los casos daremos el resultado $p$ de la prueba. Esta metodología ha sido usada recurrentemente en investigaciones en educación para encontrar diferencias relevantes entre diferentes grupos o poblaciones (Pérez, Galán y Quintal, 2012). Para el análisis de resultados se utilizó el software $\operatorname{MedCalc} \odot$, versión 16.8.4 x64. 
Este estudio dejará para futuras investigaciones si estas diferencias están relacionadas, o son determinantes de condiciones de calidad de los programas o si tienen incidencia en el desempeño de sus egresados en distintos campos -e.g. empleabilidad, resultados en pruebas estandarizadas, paso a posgrados, etc.-.

\section{Resultados}

La tabla 2 muestra el número de programas de acuerdo con la tipología que presentamos en "Introducción". El total de programas analizados es 55 , y se presenta sobre todo un número considerablemente menor de programas a distancia (9) con respecto a los presenciales (46). Es significativo, a su vez, el bajo número de aquellos con acreditación de alta calidad (17) en comparación con los que cuentan solamente con registro calificado (38).
Tabla 2. Número de programas analizados por tipología

\begin{tabular}{|l|l|l|}
\hline & & \multicolumn{1}{|c|}{$\begin{array}{c}\text { No.de } \\
\text { programas }\end{array}$} \\
\hline \multirow{2}{*}{ Título otorgado } & Profesional & 27 \\
\cline { 2 - 3 } & Licenciado & 28 \\
\hline $\begin{array}{l}\text { Especificidad } \\
\text { disciplinar }\end{array}$ & Filosofía & 37 \\
\cline { 2 - 3 } $\begin{array}{l}\text { Registro ante } \\
\text { el SNIES }\end{array}$ & Filosofía y otro & 18 \\
\hline \multirow{2}{*}{ Modalidad } & R. calificado & 38 \\
\cline { 2 - 3 } & R. alta calidad & 17 \\
\hline \multirow{2}{*}{$\begin{array}{l}\text { Sector de la } \\
\text { institución }\end{array}$} & Presencial & 46 \\
\cline { 2 - 3 } & Distancia & 9 \\
\cline { 2 - 3 } & Oficial & 21 \\
\hline
\end{tabular}

Fuente: elaboración propia.

A continuación (tabla 3 ) se presenta el resultado de aplicar las categorías establecidas en "Categorías de espacios académicos" a los planes de estudio de los cincuenta y cinco programas de pregrado en filosofía actualmente activos en Colombia:

Tabla 3. Composición de los planes de estudios de pregrado en filosofía en número de créditos

\begin{tabular}{|c|c|c|c|c|c|c|c|c|c|c|c|c|c|c|}
\hline Cód. SNIES & CF & SF & CM & LM & LC & PE & EL & DNF & PR & TIC & PRA & TG & O & Total \\
\hline 20 & 27 & 36 & 24 & 0 & 0 & 0 & 27 & 0 & 0 & 0 & 0 & 8 & 0 & 122 \\
\hline 295 & 51 & 44 & 13 & 0 & 6 & 0 & 14 & 25 & 0 & 0 & 0 & 8 & 0 & 161 \\
\hline 417 & 48 & 40 & 4 & 12 & 8 & 80 & 0 & 0 & 0 & 0 & 8 & 0 & 0 & 200 \\
\hline 441 & 48 & 40 & 4 & 12 & 8 & 0 & 0 & 0 & 60 & 0 & 0 & 0 & 0 & 172 \\
\hline 557 & 48 & 24 & 15 & 9 & 0 & 18 & 6 & 0 & 18 & 0 & 3 & 3 & 5 & 149 \\
\hline 744 & 60 & 52 & 12 & 0 & 0 & 0 & 20 & 0 & 0 & 0 & 0 & 16 & 0 & 160 \\
\hline 773 & 40 & 17 & 14 & 0 & 14 & 13 & 6 & 22 & 0 & 2 & 4 & 4 & 2 & 138 \\
\hline 933 & 55 & 23 & 24 & 8 & 3 & 0 & 10 & 6 & 12 & 0 & 0 & 7 & 0 & 148 \\
\hline 955 & 52 & 12 & 10 & 0 & 0 & 0 & 14 & 4 & 40 & 0 & 0 & 8 & 10 & 150 \\
\hline 1077 & 35 & 4 & 18 & 2 & 0 & 25 & 12 & 35 & 9 & 3 & 6 & 0 & 8 & 157 \\
\hline 1197 & 56 & 32 & 16 & 0 & 12 & 0 & 4 & 0 & 0 & 0 & 0 & 4 & 0 & 124 \\
\hline 1300 & 53 & 12 & 15 & 0 & 0 & 3 & 23 & 24 & 0 & 0 & 0 & 12 & 2 & 144 \\
\hline 1371 & 57 & 24 & 18 & 0 & 0 & 18 & 8 & 8 & 0 & 0 & 0 & 4 & 0 & 137 \\
\hline 1445 & 60 & 0 & 19 & 0 & 0 & 0 & 6 & 22 & 12 & 6 & 0 & 6 & 12 & 143 \\
\hline 1537 & 42 & 15 & 9 & 0 & 12 & 0 & 9 & 21 & 12 & 0 & 0 & 3 & 0 & 123 \\
\hline 1872 & 51 & 17 & 14 & 0 & 14 & 0 & 6 & 14 & 0 & 2 & 0 & 4 & 2 & 124 \\
\hline 2049 & 43 & 12 & 14 & 9 & 0 & 20 & 2 & 3 & 8 & 3 & 14 & 3 & 13 & 144 \\
\hline 2715 & 6 & 50 & 0 & 8 & 0 & 29 & 8 & 38 & 21 & 2 & 6 & 2 & 0 & 170 \\
\hline 2718 & 34 & 16 & 2 & 0 & 4 & 20 & 4 & 40 & 28 & 0 & 4 & 4 & 4 & 160 \\
\hline 4074 & 57 & 12 & 24 & 12 & 0 & 0 & 3 & 15 & 21 & 3 & 0 & 0 & 3 & 150 \\
\hline 4378 & 21 & 9 & 12 & 9 & 0 & 31 & 4 & 20 & 21 & 3 & 8 & 4 & 14 & 156 \\
\hline 4450 & 35 & 12 & 13 & 8 & 4 & 23 & 0 & 10 & 40 & 3 & 6 & 4 & 2 & 160 \\
\hline 8167 & 44 & 12 & 22 & 15 & 6 & 0 & 10 & 21 & 20 & 0 & 0 & 4 & 1 & 155 \\
\hline 8460 & 85 & 0 & 12 & 8 & 0 & 0 & 17 & 0 & 0 & 0 & 0 & 10 & 3 & 135 \\
\hline 9363 & 52 & 20 & 17 & 9 & 6 & 0 & 2 & 3 & 8 & 3 & 11 & 3 & 10 & 144 \\
\hline 9570 & 30 & 0 & 15 & 0 & 0 & 50 & 9 & 23 & 0 & 0 & 12 & 0 & 0 & 139 \\
\hline
\end{tabular}




\begin{tabular}{|c|c|c|c|c|c|c|c|c|c|c|c|c|c|c|}
\hline Cód. SNIES & CF & SF & CM & LM & LC & PE & EL & DNF & PR & TIC & PRA & TG & 0 & Total \\
\hline 9693 & 59 & 26 & 12 & 0 & 0 & 0 & 6 & 6 & 20 & 0 & 0 & 4 & 3 & 136 \\
\hline 10264 & 55 & 40 & 9 & 0 & 8 & 12 & 0 & 7 & 16 & 0 & 11 & 6 & 0 & 164 \\
\hline 10463 & 44 & 40 & 16 & 0 & 3 & 20 & 16 & 6 & 12 & 3 & 9 & 0 & 3 & 172 \\
\hline 10793 & 35 & 2 & 33 & 6 & 0 & 16 & 18 & 21 & 9 & 2 & 6 & 0 & 9 & 157 \\
\hline 10798 & 35 & 2 & 33 & 6 & 0 & 16 & 18 & 21 & 9 & 2 & 6 & 0 & 9 & 157 \\
\hline 11374 & 48 & 18 & 15 & 9 & 0 & 0 & 9 & 0 & 12 & 0 & 0 & 7 & 5 & 123 \\
\hline 11927 & 72 & 28 & 2 & 0 & 8 & 0 & 0 & 10 & 24 & 0 & 0 & 16 & 0 & 160 \\
\hline 11929 & 38 & 0 & 14 & 0 & 0 & 0 & 0 & 100 & 0 & 0 & 3 & 6 & 5 & 166 \\
\hline 11967 & 88 & 32 & 24 & 0 & 0 & 0 & 20 & 0 & 0 & 0 & 0 & 10 & 0 & 174 \\
\hline 11971 & 86 & 6 & 14 & 12 & 8 & 0 & 8 & 2 & 0 & 2 & 0 & 4 & 2 & 144 \\
\hline 12047 & 12 & 60 & 42 & 0 & 7 & 0 & 3 & 3 & 0 & 0 & 7 & 4 & 32 & 170 \\
\hline 13217 & 40 & 0 & 15 & 4 & 0 & 19 & 12 & 38 & 16 & 0 & 5 & 6 & 3 & 158 \\
\hline 13218 & 35 & 24 & 16 & 0 & 6 & 21 & 9 & 27 & 0 & 0 & 15 & 8 & 0 & 161 \\
\hline 14826 & 45 & 27 & 15 & 8 & 4 & 0 & 12 & 0 & 12 & 0 & 4 & 8 & 8 & 143 \\
\hline 15235 & 31 & 2 & 15 & 12 & 0 & 57 & 2 & 38 & 2 & 0 & 8 & 0 & 0 & 167 \\
\hline 20444 & 48 & 6 & 12 & 12 & 0 & 28 & 12 & 4 & 0 & 4 & 12 & 4 & 2 & 144 \\
\hline 20650 & 48 & 6 & 12 & 12 & 0 & 28 & 12 & 4 & 0 & 4 & 12 & 4 & 2 & 144 \\
\hline 53049 & 85 & 10 & 18 & 0 & 0 & 0 & 8 & 15 & 0 & 0 & 0 & 8 & 0 & 144 \\
\hline 53718 & 54 & 30 & 15 & 0 & 6 & 25 & 8 & 0 & 0 & 4 & 12 & 6 & 0 & 160 \\
\hline 53758 & 86 & 6 & 14 & 12 & 8 & 0 & 8 & 2 & 0 & 2 & 0 & 4 & 2 & 144 \\
\hline 54163 & 56 & 12 & 8 & 0 & 0 & 28 & 14 & 0 & 40 & 0 & 4 & 10 & 14 & 186 \\
\hline 54345 & 59 & 7 & 18 & 0 & 8 & 18 & 0 & 6 & 0 & 0 & 7 & 3 & 2 & 128 \\
\hline 90479 & 52 & 0 & 12 & 0 & 0 & 30 & 18 & 24 & 0 & 0 & 4 & 5 & 5 & 150 \\
\hline 90553 & 48 & 40 & 4 & 12 & 8 & 80 & 0 & 0 & 0 & 0 & 8 & 0 & 0 & 200 \\
\hline 90806 & 51 & 30 & 13 & 20 & 8 & 0 & 9 & 0 & 12 & 3 & 0 & 9 & 8 & 163 \\
\hline 91044 & 31 & 3 & 16 & 0 & 4 & 39 & 8 & 14 & 16 & 0 & 14 & 0 & 10 & 155 \\
\hline 91267 & 46 & 40 & 9 & 9 & 6 & 80 & 0 & 0 & 0 & 0 & 10 & 0 & 0 & 200 \\
\hline 103785 & 33 & 21 & 15 & 16 & 0 & 0 & 6 & 39 & 0 & 0 & 0 & 6 & 0 & 136 \\
\hline 105065 & 30 & 18 & 15 & 0 & 0 & 36 & 6 & 24 & 0 & 0 & 3 & 3 & 0 & 135 \\
\hline
\end{tabular}

Fuente: elaboración propia.

\section{Análisis de resultados}

\section{Media de créditos por categoría para todos los programas}

En la tabla 4 se presentan las medias para todos los programas incluidos en el estudio. De acuerdo con esta, los programas de filosofía en Colombia tienen un alto contenido de CF $(48,00 \mathrm{cr}$.) y SF $(19,47$ cr.), que, junto con los см $(14,84 \mathrm{cr}$.) cubren la mayoría de los planes de estudio $(82,31 \mathrm{cr}$.), teniendo en cuenta que el promedio es de 152,84. Resulta significativo que el promedio de créditos de DNF $(13,91 \mathrm{cr}$.) y el de PE $(16,05 \mathrm{cr}$.) sean cercanos a los de SF o CM, ya que muchos programas no contemplan estas categorías, como se puede ver en la tabla 3. Para poder hacer un análisis más detallado de estos valores abordaremos las medias de los programas según las tipologías, pues, como veremos más adelante, los promedios varían en función de estas.

Tabla 4. Media de créditos por categoría de espacio académico para todos los programas

\begin{tabular}{|c|c|c|c|c|c|c|c|c|c|c|c|c|c|}
\hline & CF & SF & CM & LM & LC & PE & EL & DNF & PR & TIC & PRA & TG & 0 \\
\hline Media & 48,00 & 19,47 & 14,84 & 4,93 & 3,44 & 16,05 & 8,47 & 13,91 & 9,64 & 1,02 & 4,40 & 4,76 & 3,91 \\
\hline
\end{tabular}

Fuente: elaboración propia. 


\section{Análisis por tipología de programas}

Al clasificar los programas en función del título otorgado, como vemos en la tabla 5 , se evidencian diferencias de la composición de los programas. Los programas profesionales tienden a tener un mayor número de créditos en cursos filosóficos $(55,41$ contra 40,86), mientras las licenciaturas tienen considerablemente más cursos de pedagogía que los profesionales $(31,43 \mathrm{cr}$. contra $0,11 \mathrm{cr}$ ) y prácticas $(7,75 \mathrm{cr}$. contra $0,93 \mathrm{cr}$.). Valga señalar que, aunque son escasos, hay programas profesionales que tienen tanto materias pedagógicas (código SNIES 1300) como prácticas (códigos SNIEs 9363, 11929, 12047, 14826). También es notorio que el número de créditos destinado al trabajo de grado en las licenciaturas $(2,96$ cr. $)$ es en promedio menos de la mitad que en los programas profesionales $(6,63 \mathrm{cr}$.).

Al comparar los programas de filosofía contra los programas de filosofía y otra disciplina (tabla 6), si bien hay varios programas filosóficos que tienen asignaturas disciplinares no filosóficas, en general en los programas que combinan filosofía con otra disciplina tienen una media de créditos mucho mayor $(31,17$ contra 5,51). Es notorio también que, probablemente a causa de lo anterior, en los que combinan filosofía con otra disciplina se reduce considerablemente el número de créditos en cursos filosóficos $(54,16$ contra 35,33$)$ y de seminarios de filosofía $(22,76$ contra 12,72 ). Aumenta ligeramente (aunque no de manera significativa) el número de créditos de prácticas $(3,84$ contra 5,56), debido, tal vez, a que hay más licenciaturas en este tipo de programas (13 licenciaturas, 5 profesionales), mientras que los que son exclusivamente en filosofía la proporción de licenciaturas es menor (15 licenciaturas, 22 profesionales).

Tal como lo muestra la tabla 7 , no son estadísticamente significativas las diferencias entre los programas si se clasifican por tipo de registro, especialmente en cursos de filosofía, metodológicos, electivas libres, cursos de profundización, TIC o trabajos de grado. Hay unas ligeras diferencias (aunque no estadísticamente significativas) en los seminarios de filosofía, en el que los programas que solo tienen registro cuentan con una media más baja $(18,05$ contra 22,65). También los créditos en lenguas clásicas son más bajos para estos programas $(2,66$ contra 5,18). La diferencia más notoria (aunque no es estadísticamente significativa) puede estar en las asignaturas pedagógicas, que son mayores para los programas con registro calificado que para los que cuentan con alta calidad (18,42 contra 10,76). Es probable que esta diferencia se deba a que hay mayor cantidad de licenciaturas que solo cuentan con registro calificado (tabla 2); esto lo veremos con más detalle en "Licenciaturas en filosofía".

Al clasificar los programas por modalidad hay varias diferencias significativas. Es muy superior el número de créditos en seminarios que tienen los programas presenciales contra los que son a distancia $(22,28$ contra 5,11). Esta es una de las mayores diferencias que se han presentado hasta el momento entre los componentes de los planes de estudios y tal vez se deba a que en modalidad a distancia es bastante difícil implementar cursos tipo seminario en los que las intervenciones de los estudiantes, la participación y el debate son mayores que en un curso magistral. También es significativo el mayor número de créditos en trabajo de grado para los programas presenciales $(5,43$ contra 1,33$)$, posiblemente en virtud de las distintas modalidades de trabajo de grado en los programas a distancia. En contraposición, es significativo el menor número de créditos de los programas presenciales en cursos metodológicos con respecto a los que son a distancia (13,89 contra 19,67), lo que puede deberse a que los estudiantes de los programas a distancia, por lo general, tienen menores habilidades comunicativas que los programas presenciales (Escané, Herrero, Merlino y Ayllón, 2014) y con este énfasis en el componente metodológico se busca nivelar esta desventaja. También es significativo la menor cifra de créditos en lenguas modernas $(4,20$ contra 7,78$)$ y de TIC $(0,84$ contra 2,11 ), probablemente debido a que los programas a distancia requieren mayor uso de la tecnología y, en consonancia con esto, un mejor dominio de inglés. Ligeramente inferior, aunque no estadísticamente significativo -si bien $p$ está bastante cerca del $\alpha$-, es el número de créditos en lenguas clásicas $(3,85$ en programas presenciales contra 1,33 en programas a 

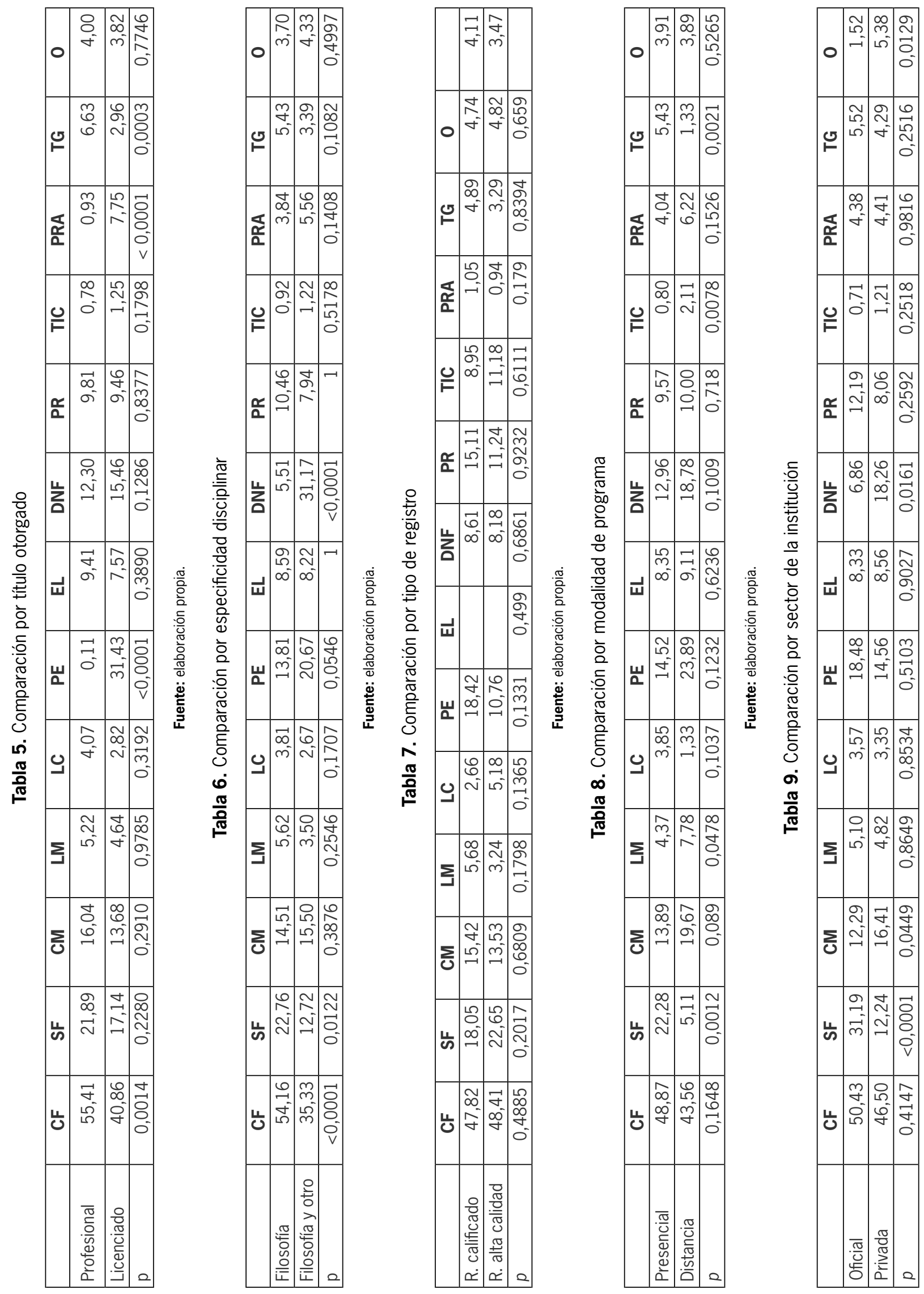

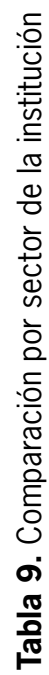

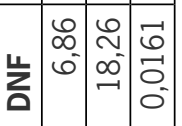


distancia). Es difícil arrojar una hipótesis que explique esta diferencia, pues la modalidad no parece ser un impedimento para la enseñanza de lenguas clásicas. Tampoco podría ser por el tipo de universidad, pues solamente dos programas (códigos SNIEs 4450 y 53758) cuentan con cursos en lenguas clásicas; uno en universidad pública, el otro en privada confesional; uno de ellos, licenciatura; el otro, profesional. Tal vez se deba a las características propias de los programas en sus énfasis disciplinares, pues es de suponer que los programas con cursos en lenguas clásicas hacen más énfasis en historia de la filosofía.

Con respecto al sector al que pertenece la institución, es considerablemente mayor el número de créditos en seminarios (31,19 contra 12,24). Esto muestra que en las instituciones privadas tienden a preferirse otras modalidades de cursos que el seminario. También es probable que se deba a que los programas de filosofía de universidades privadas cuenten con menos estudiantes y que los cursos sean en general más pequeños, lo que explicaría que muchos de sus cursos fueran impartidos en modalidad de seminario, aunque este rótulo no aparezca explícito en el plan de estudios. En las universidades públicas, en contraposición, los cursos tienden a ser más grandes y se requiere una distinción más explícita entre curso y seminario; sin embargo, esto es una mera hipótesis que requeriría un estudio más detallado para poder verificarla. También es superior el número de créditos en cursos de profundización $(12,19$ contra 8,06$)$ y trabajo de grado $(5,52$ contra 4,29), aunque no son diferencias estadísticamente significativas, lo que probablemente implica una mayor especialización de los estudiantes en los programas de instituciones oficiales, aunque esto de igual manera requeriría un estudio más profundo para poderlo verificar. En contraste, los programas de instituciones privadas son superiores en promedio en número de créditos dedicados a cursos metodológicos (16,41 contra 12,29), lo que implica una mayor concentración en competencias genéricas y transversales, aunque podría deberse a que los estudiantes de algunas universidades privadas ingresan con mayores necesidades en competencias básicas, lo que obliga a estas instituciones a tener más cursos de este tipo; aunque esto requeriría un estudio más detallado. Además, también es superior el número de créditos de asignaturas disciplinares no filosóficas $(18,26$ contra 6,86$)$, lo que muestra que los programas de instituciones privadas tienden a ser mucho más interdisciplinares; de hecho, 13 de los 16 programas cuyo título es en filosofía y otra disciplina son en universidades privadas, mientras que en las públicas solamente hay 3 programas de este tipo. También es considerablemente superior el número de otras asignaturas (5,38 contra 1,52$)$; esto porque en general casi todas las instituciones privadas cuentan con cátedras institucionales y asignaturas obligatorias para todos sus programas, mientras que en las instituciones públicas estas asignaturas son más bien escasas.

\section{Programas profesionales en filosofía}

A continuación, examinaremos solamente los programas profesionales en filosofía de acuerdo con las demás tipologías de programas. El número total de programas es de 26 , y se clasifican como lo muestra la tabla 10.

Tabla 10. Número de programas profesionales en filosofía por tipo

\begin{tabular}{|l|l|l|}
\hline \multicolumn{2}{|c|}{} & \multicolumn{1}{c|}{ Programas } \\
\hline $\begin{array}{l}\text { Especificidad } \\
\text { disciplinar }\end{array}$ & Filosofía & 22 \\
\cline { 2 - 3 } $\begin{array}{l}\text { Registro ante } \\
\text { el SNIES }\end{array}$ & Filosofía y otro & 5 \\
\hline \multirow{2}{*}{ Modalidad } & R. calificado & 17 \\
\cline { 2 - 3 } & R. alta calidad & 10 \\
\hline \multirow{2}{*}{ Tipo de institución } & Presencial & 25 \\
\cline { 2 - 3 } & Distancia & 2 \\
\cline { 2 - 3 } & Oficial & 11 \\
\cline { 2 - 3 } & Privada & 16 \\
\hline
\end{tabular}

Fuente: elaboración propia.

Es considerablemente mayor el número de programas de filosofía (22) en comparación con los que combinan filosofía con otras disciplinas (4). También es mayor el número de programas presenciales (24) con respecto a los que son a distancia (2). Como veremos a continuación, estas características de los programas implican a su vez significativas diferencias entre la composición de los planes de estudios. Como era predecible, los programas que combinan filosofía con otras disciplinas (códigos SNIES 295, 
$1445,1872,11929,103785)$ tienen en promedio un número de créditos significativamente mayor en cursos disciplinares no filosóficos (40 contra 6). Este énfasis en las asignaturas disciplinares no filosóficas puede explicar la diferencia en otros tipos de cursos, pues, aunque no se trate de diferencias estadísticamente significativas, en este tipo de programas se reducen ligeramente el número de créditos en seminarios (16,40 contra 23,14), electivas libres (6,40 contra 10,09), profundización ( 2,40 contra 11,50$)$. Solo uno de estos programas incluye créditos en lenguas modernas (código SNIEs 103785), lo que supone que, si las demás instituciones exigen requisito de idioma para grado, utilizan una estrategia distinta a la inclusión de clases en el plan de estudios, tal como lo contempla el Decreto 1075 de 2015 (MEN, art. 2.5.3.2.2.1; $\$ 3.8$ ). Algunas universidades cuentan con cursos de idiomas independientes del plan de estudios, pero de obligatorio cumplimiento para los estudiantes como requisito de grado, aunque en algunos casos estos cursos se pueden aprobar por medio de un examen de suficiencia. Otras simplemente exigen certificados internacionales de idiomas.

Entre los programas que cuentan con registro calificado y los que presentan acreditación de alta calidad, hay diferencias significativas a favor de los programas que solo cuentan con registro en el número de asignaturas en lenguas modernas (7,06 contra 2,10). Hay otras diferencias menores, no estadísticamente significativas, en cursos filosóficos $(59,06$ contra 49,20), metodológicos $(17,18$ contra $14,10)$ y trabajo de grado $(7,24$ contra 5,60$)$. Si tenemos en cuenta que esta clasificación supone una diferenciación de calidad considerable entre los programas estudiados -en principio los programas con acreditación de alta calidad deberían ser mejores que los que solamente cuentan con registro calificado-, podemos ver que hay elementos de la composición del plan de estudios que no son determinantes para una acreditación de calidad para un programa; sobre todo aquellos en los que los programas con registro son superiores a los de alta calidad. Este parece ser el caso de lenguas modernas, lo que supone que los programas con acreditación de alta calidad utilizan otras estrategias pedagógicas y curriculares para fomentar la formación en otras lenguas. Sin duda, esto requiere un estudio más profundo para examinar si hay algún tipo de impacto entre el número de créditos de los programas o las estrategias curriculares utilizadas con respecto al desempeño de los estudiantes en lenguas extranjeras. Sucede lo contrario en créditos consagrados a lenguas clásicas, en donde los programas con registro calificado son inferiores a los de acreditación (3,06 contra 5,80), electivas libres $(8,76$ contra 10,50$)$ y profundización $(7,82$ contra 13,20$)$; esta última evidencia una diferencia de calidad entre los dos tipos de programas, al preferir los programas con acreditación un mayor énfasis y especialización de sus estudiantes a diferencia de los programas que solamente cuentan con registro. El porcentaje mayor de electivas libres para los programas de alta calidad también supone mayor flexibilidad, lo que concuerda con lo concluido en investigaciones anteriores (Farieta, Gómez y Almeida, 2015).

De acuerdo con la modalidad del programa hay considerables diferencias en la composición de los planes de estudios entre los programas presenciales y los que son a distancia. La mayor diferencia tiene que ver con el uso de TIC ( 0,64 contra 2,50), que es obligatoria en los programas a distancia (Decreto 1075 de 2015, art. 2.5.3.2.2.1; \$7.1.4).

Hay otras diferencias no estadísticamente significativas, pero notorias, con respecto a los cursos filosóficos $(54,12$ contra 71,50$)$ y los seminarios $(22,92$ contra 9,00). Esto supone que los programas a distancia prefieren en general los cursos sobre los seminarios, presumiblemente porque puede ser bastante difícil llevar a cabo una asignatura en modalidad seminario en los programas que son a distancia, tal como lo dijimos anteriormente ( $c f$. tabla 8). Podría suceder también que algunos de los cursos se llevasen a cabo en modalidad seminario, tal como consideramos que puede ocurrir con algunos cursos de universidades privadas ( $c f$. tabla 9); sin embargo, en general en los programas a distancia, contrariamente a lo que sucede en los programas de instituciones privadas, el número de estudiantes por curso suele ser mayor que en cursos presenciales (Vanegas, Valencia, 


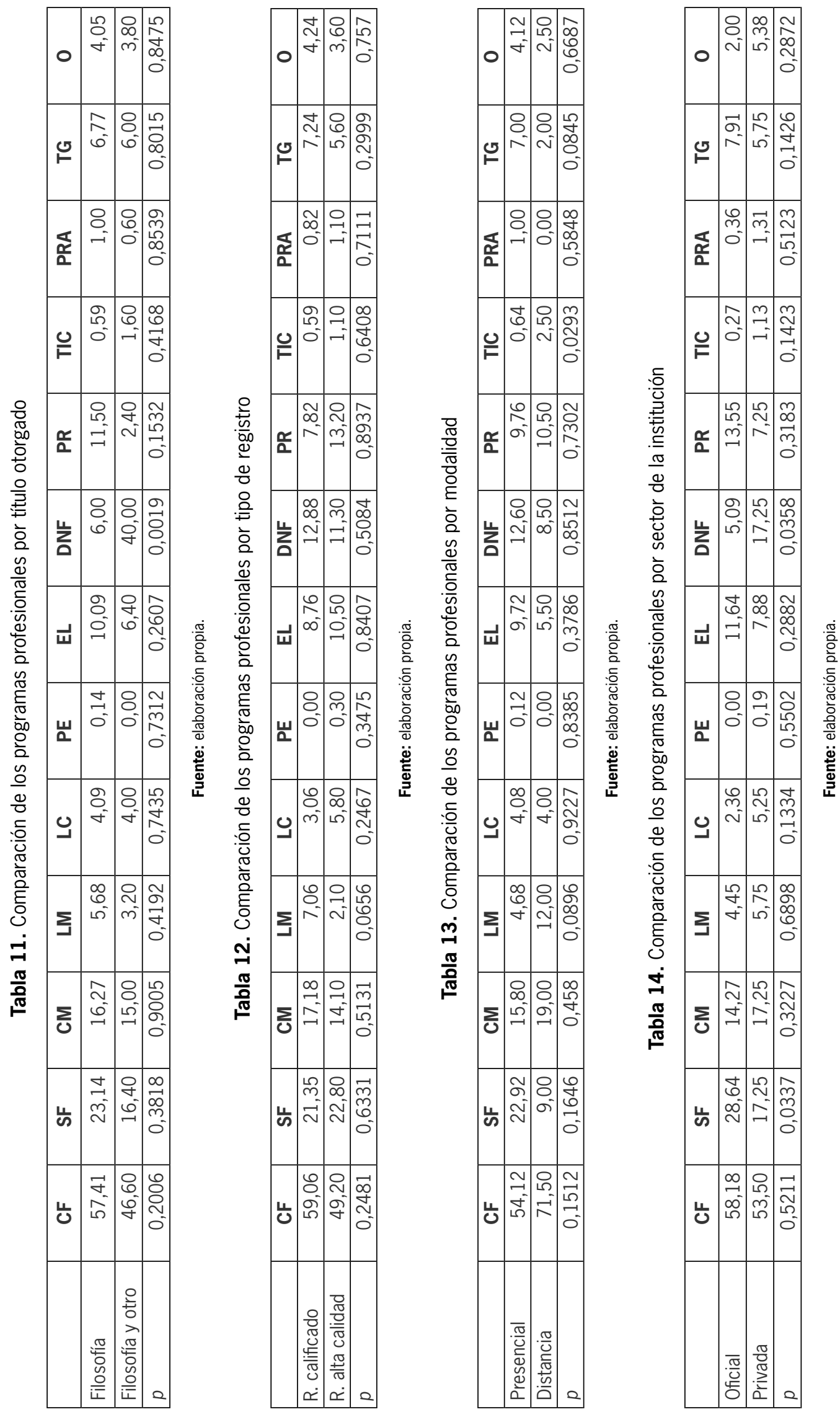


Reinoso y Cortés, 2013). Es notorio, aunque no estadísticamente significativo, el menor número de cursos metodológicos para los programas presenciales (15,80 contra 19$)$; es probable que los programas a distancia reciban estudiantes con mayores necesidades en sus habilidades de lectura y escritura, lo que podría sustentar la preferencia por este tipo de cursos (Escanés et al., 2014), pero esto requeriría estudios más detallados. Es bastante significativa la diferencia en el número de créditos en lenguas modernas (4,68 contra 12), lo que implica que hay un mayor énfasis en bilingüismo en los programas a distancia. En cambio, el número de créditos en electivas libres disminuye $(9,72$ contra 5,50$)$, lo que muestra que los programas a distancia son menos flexibles y sus estudiantes interactúan menos con estudiantes de otras disciplinas. Por último, también es menor el número de créditos en trabajo de grado de los programas a distancia $(2,00$ contra 7,00$)$, lo que parece indicar que este tipo de programas toman el trabajo de grado como una materia más del plan de estudios que se lleva a cabo solo en un semestre y no representa una carga significativa en el plan de estudios.

De acuerdo con el sector de la institución, la diferencia más significativa es que los programas profesionales de universidades oficiales contienen muchos más créditos en seminarios en promedio que los programas de universidades privadas $(28,64$ contra 17,25$)$, lo que está acorde con la explicación que dimos anteriormente para todos los programas de filosofía (cf. tabla 9). Es significativamente superior en las universidades privadas el número de créditos en asignaturas disciplinares no filosóficas $(17,25$ contra 5,09), debido, tal vez, a que hay más programas profesionales que combinan filosofía con otras disciplinas en las universidades privadas (3 programas) que en las públicas (un solo programa). Sin embargo, también parece que las instituciones privadas tienen más componente interdisciplinar que las públicas, como presentamos antes ( $c f$. tabla 9). Aunque, por otra parte, las instituciones oficiales tienen más créditos de profundización (13,55 contra 7,25$)$ y trabajo de grado $(7,91$ contra 5,75$)$, por lo que estas parecen preferir una mayor especialización disciplinar. También las instituciones privadas tienden a tener mayor componente de asignaturas metodológicas (17,25 contra 14,27), aunque la diferencia no es estadísticamente significativa; esto se podría explicar en función de que las universidades oficiales cuentan con un número reducido de cupos para estudiantes y llevan a cabo exámenes de admisión para seleccionar a quienes ingresan; esto no necesariamente sucede en las universidades privadas, que tienen procesos menos exigentes de admisión; aunque las desventajas que traen estos estudiantes en aspectos metodológicos - principalmente de lectura y escritura- son compensadas o niveladas por la universidad con este tipo de cursos. También es notoria la ligera superioridad de créditos en las universidades privadas en lenguas clásicas (2,36 contra $5,25)$. Esto se puede deber a que, dado que varias de las universidades son confesionales y forman a sacerdotes o religiosos, tienen que impartir formación en lenguas clásicas por directriz del Vaticano (Pablo VI, 1965). Por último, el número de créditos en TIC en las públicas es muy bajo en comparación con las privadas $(0,27$ contra 1,31$)$, aunque la diferencia no es estadísticamente significativa. Si bien en general el promedio es bajo, se resalta que en los programas de las públicas en general es muy escaso este tipo de espacios académicos -solo en un programa se ofrece una asignatura, y es un programa a distancia (código sNies 4074), lo que explicaría su obligatoriedad-, o puede suceder que sean parte del componente electivo de los programas, pero que no sea obligatorio para todos los estudiantes.

\section{Licenciaturas en filosofía}

A continuación, examinaremos las licenciaturas en filosofía activas en Colombia; un total de 28, que se pueden clasificar como se presentan en la tabla 15 con base en lo presentado en la introducción. 
Universidad Pedagógica Nacional

Facultad de Humanidades

Tabla 15. Número de licenciaturas en filosofía por tipo

\begin{tabular}{|l|l|r|}
\hline & & Programas \\
\hline \multirow{2}{*}{$\begin{array}{l}\text { Especificidad } \\
\text { disciplinar }\end{array}$} & Filosofía & 15 \\
\cline { 2 - 3 } $\begin{array}{l}\text { Registro ante } \\
\text { el SNIES }\end{array}$ & Filosofía y otro & 13 \\
\hline \multirow{2}{*}{ Modalidad } & R. calificado & 21 \\
\cline { 2 - 3 } & Presencial & 7 \\
\cline { 2 - 3 } & Distancia & 7 \\
\hline \multirow{2}{*}{ Tipo de institución } & Oficial & 10 \\
\cline { 2 - 3 } & Privada & 18 \\
\hline
\end{tabular}

Fuente: elaboración propia.

En comparación con los programas profesionales (tabla 10), hay más licenciaturas que combinan filosofía con otras disciplinas (13 lic. contra 4 prof.), por lo que en general son más interdisciplinares que los programas profesionales. Las licenciaturas que cuentan con registro de alta calidad (7) son menos que las que cuentan con registro calificado (21), y a su vez menos que los programas profesionales con alta calidad (10). También hay más licenciaturas a distancia (7) que programas profesionales (2). Por último, hay menos licenciaturas en instituciones oficiales (10) que programas profesionales (11); mientras que hay más licenciaturas en instituciones privadas (18) que programas profesionales en este mismo tipo de instituciones (16).

Tal como sucedía con los programas profesionales (cf. tabla 11), las licenciaturas que combinan filosofía con otras disciplinas tienen significativamente más créditos en asignaturas disciplinares no filosóficas $(27,77)$ contra las licenciaturas que son solo de filosofía $(4,80)$; esto explica que en casi todos los demás tipos de espacios académicos la relación sea inversa. Así, en las licenciaturas que son solamente en filosofía aumenta significativamente el número de créditos en cursos $(49,40$ contra 31,00$)$ y de seminarios $(22,20$ contra 11,31$)$. También hay un aumento, aunque no estadísticamente significativo, en cursos de lengua moderna $(5,53$ contra 3,62$)$ de asignaturas pedagógicas $(33,87$ contra 28,62$)$, y de trabajo de grado $(3,47$ contra 2,38). Por el contrario, para las licenciaturas que son solamente en filosofía es ligeramente inferior el número de créditos en electivas libres $(6,40$ contra 8,92$)$ y de profundización
$(8,93$ contra 10,08$)$-contrario a lo que sucede entre los programas profesionales ( $c f$. tabla 11)-, lo que muestra que los programas que incluyen más disciplinas tienden a ser más flexibles en su componente de profundización, presumiblemente porque el estudiante puede escoger entre diferentes énfasis disciplinares del programa. Vale la pena resaltar también el menor número de créditos de componente metodológico en las licenciaturas solamente en filosofía (11,93 contra 15,75), quizás en virtud de que las licenciaturas que combinan filosofía con otras disciplinas requieren estudiar las metodologías de investigación de estas otras disciplinas.

De acuerdo con el tipo de registro, los programas que solamente cuentan con registro calificado tienen un significativo número mayor de créditos en asignaturas pedagógicas (33,33 contra 25,71), lo que parece evidenciar un mayor énfasis en el carácter pedagógico y educativo de estos programas. También es mayor, aunque no estadísticamente significativo, el número de créditos en electivas libres ( 8,48 contra 4,86 ), lo que indica un promedio relativamente bajo de electivas libres para programas con acreditación de alta calidad. Pese a esto, estos programas tal vez aplican su flexibilidad en otros componentes optativos disciplinares y no solamente en las electivas libres, pues en general el porcentaje de flexibilidad es mayor en los programas con acreditación de alta calidad (Farieta, Gómez y Almeida, 2015). En contraste, en los programas con registro calificado es significativamente menor el número de créditos en cursos filosóficos (38,71 contra 47,29), también son menores, aunque poco significativos, los créditos en seminarios filosóficos $(15,38$ contra 22,43$)$ y trabajo de grado (2,71 contra 3,71$)$, lo que parece indicar un mayor énfasis disciplinar de los programas con acreditación de alta calidad. También es mayor el número de asignaturas disciplinares no filosóficas con respecto a los programas de alta calidad (16,90 contra 11,14), lo cual se debe a que en general son mucho más las licenciaturas con registro calificado que combinan filosofía con otras disciplinas (11 de 21 licenciaturas con r. calificado) que las que cuentan con alta calidad y combinan varias disciplinas ( 2 de 7 licenciaturas con $r$. alta calidad). 
Siguiendo con la tendencia general ( $c f$. tabla 8$)$ y la de los programas profesionales ( $c f$. tabla 13), las licenciaturas presenciales tienen en promedio muchos más créditos en seminarios filosóficos que las virtuales $(21,52$ contra 4,00$)$. Como ya mencionamos, tal vez la razón es la dificultad de implementar esta modalidad de curso en un programa a distancia o virtual. También es significativamente mayor el número de créditos en cursos filosóficos $(42,62$ contra 35,57$)$ y lenguas clásicas ( 3,57 contra 0,57$)$; solo una licenciatura ofrece este tipo de cursos (código sNIEs 4450). Notorio, aunque no estadísticamente significativo, es que la proporción de cursos disciplinares no filosóficos es menor en los programas presenciales $(13,38$ contra 21,71). Esto puede deberse a que la mayoría de licenciaturas a distancia combinan filosofía con otras disciplinas ( 5 de 7), lo que hace que el componente interdisciplinar sea mayor en estos programas y sean menores los componentes disciplinares en cursos y seminarios filosóficos. Por otra parte, es significativo el menor número de créditos en asignaturas metodológicas en los programas presenciales $(11,62$ contra 19,86). Esto también puede deberse a que más licenciaturas a distancia combinan filosofía con otras disciplinas, lo que requiere estudiar las metodologías de investigación propias de las otras disciplinas que componen los programas, aunque esto requeriría un estudio más detallado.

De acuerdo con el sector de la institución también hay diferencias significativas entre las licenciaturas en Colombia. La mayor de ellas se presenta en el número de créditos dedicados a seminarios filosóficos, en donde las instituciones oficiales superan por un muy amplio margen $(34,00$ contra 7,78$)$, mayor que el que presenta el promedio general de los programas ( $c f$. tabla 9) y de los programas profesionales ( $c f$. tabla 14). También es mayor el promedio de número de créditos en lenguas clásicas (4,90 contra 1,76), tal vez debido a que los programas de instituciones oficiales hacen más énfasis en historia de la filosofía y exigen de sus estudiantes una mayor profundización en cursos de historia de la filosofía antigua y medieval que requieren estudiar además lenguas clásicas como el griego y el latín; si bien en los programas profesionales la relación es inversa.
También es probable que las instituciones privadas sacrifiquen las lenguas clásicas en sus licenciaturas para dar más espacio a materias pedagógicas, sobre todo si tenemos en cuenta que las licenciaturas de universidades privadas son más cortas que las de las oficiales (Farieta, Gómez y Almeida, 2015). Es mayor el número de créditos en electivas libres para las universidades privadas, lo que muestra una relación inversa a la que se da entre los programas profesionales ( $c f$. tabla 14). Esto muestra que hay también diferencias importantes entre los programas profesionales y las licenciaturas en las universidades oficiales, sobre todo si se tiene en cuenta que solamente en cuatro de estas instituciones hay doble programa (profesional y licenciatura), mientras que en ocho de las privadas hay doble programa.

También es menor el número de créditos en asignaturas disciplinares no filosóficas $(8,80$. contra $19,17)$. Esto en virtud de que mientras en las públicas solamente hay dos licenciaturas que combinan filosofía con otras disciplinas (códigos sNIEs 2715 y 13218), en 11 de las 17 licenciaturas de universidades privadas se combinan filosofía con otra disciplina. También es menor el número de créditos en otras asignaturas ( 1 contra 5,39), pues, tal como sucede en general ( $c f$. tabla 9) y con los programas profesionales ( $c f$. tabla 14), las instituciones privadas exigen a sus programas una serie de cursos o cátedras institucionales que no tienen lugar en las instituciones oficiales.

\section{Conclusiones}

\section{Caracterización de los planes de estudio de filosofía en Colombia}

Hemos presentado en detalle una caracterización de la composición de los planes de estudio de filosofía en Colombia, señalando sus principales diferencias según la tipología de programas. Con respecto a esta, podemos concluir, corroborando lo señalado en estudios anteriores (Farieta, Gómez y Almeida, 2015) que las principales diferencias se encuentran entre los programas de universidades oficiales con respecto a los de universidades privadas ( $c f$. tabla 9); las diferencias son más marcadas entre las licenciaturas 


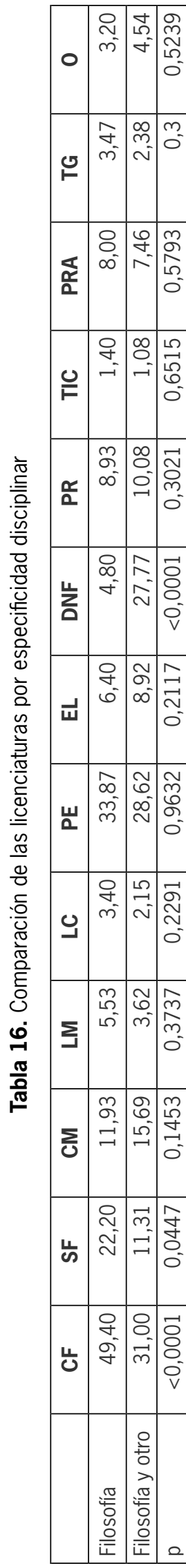

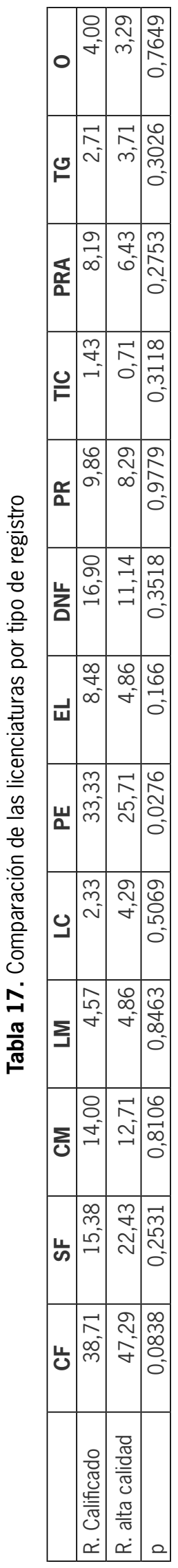
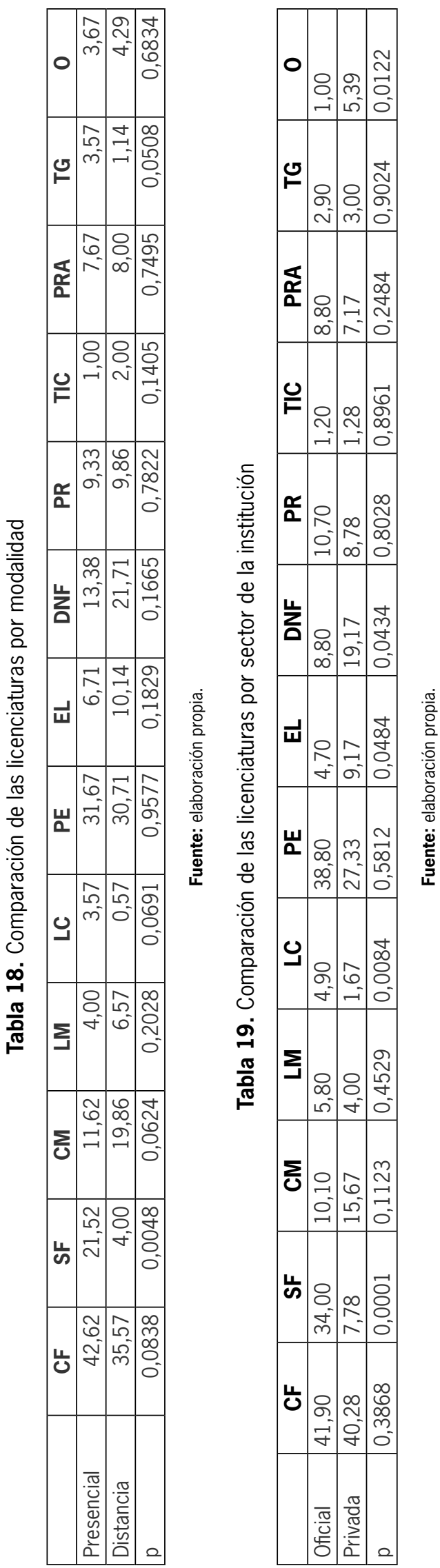
( $c f$. tabla 19), en donde se encontraron cinco diferencias estadísticamente significativas; el mayor número de las comparaciones realizadas. Estas diferencias pueden ser la consecuencia también de tendencias ideológicas de las universidades privadas, las cuales en su mayoría son de carácter religioso -más específicamente católico- o dependen o están fundamentadas en el humanismo cristiano; sobre todo en las licenciaturas.

También, y en conformidad con el estudio anterior (Farieta, Gómez y Almeida, 2015), se encontraron bastantes diferencias estadísticamente entre los programas profesionales y las licenciaturas ( $c f$. tabla 5); sobre todo entre las universidades públicas y privadas ( $c f$. tabla 19). Dado que el cálculo se hizo en términos de número de créditos y no en porcentaje con respecto al total del programa, vale la pena señalar que estas diferencias no deberían estar asociadas directamente con la diferencia en duración que hay entre programa profesional y licenciatura, pues en general las licenciaturas son más largas y tienen más créditos que los programas profesionales; aunque las licenciaturas de universidades privadas son más cortas y tienen una duración similar a los programas profesionales de universidades privadas (Farieta, Gómez y Almeida, 2015).

Lo que muestra el estudio, entonces, es que las licenciaturas que tienen la misma duración que los programas disciplinares tienden a sacrificar diferentes áreas de estudio -tanto disciplinares como complementarias, como lenguas- en pro de tener programas más cortos. A partir de estas diferencias, queda como una propuesta importante examinar si estas diferencias en la duración de los programas o en los contenidos de los mismos tienen implicaciones en el desempeño de los estudiantes. Para un futuro estudio proponemos entonces hacer una evaluación de estas características de los programas con base en indicadores de desempeño (pruebas estandarizadas, empleabilidad, salario, paso a posgrados, etc.). Una diferencia también muy marcada se encuentra entre los programas presenciales y a distancia ( $c f$. tabla 8); más marcada en las licenciaturas ( $c f$. tabla 18) que en los profesionales, diferencias en los principales componentes de los planes de estudios, como los seminarios, los cursos metodológicos y trabajo de grado. Es necesario tener en cuenta estas diferencias para futuros estudios de evaluación de desempeño, porque podría ser difícil determinar si la diferencia de desempeño entre ambos tipos de programas se deba o bien a la modalidad misma del programa o a las características de la composición del programa, más que a la modalidad misma. Para encontrar resultados acertados valdría la pena separar la variable de la modalidad con respecto a la de los componentes del programa.

Por último, hay que resaltar que fueron muy pocas las diferencias que se hallaron entre los programas con registro calificado y los que tienen acreditación de alta calidad. Si tenemos en cuenta que esta tipología clasifica los programas en función de criterios objetivos de calidad, y que los que tienen acreditación de alta calidad son considerados programas de excelencia académica por el CNA, podemos concluir que la estructura de la malla curricular no ha sido un criterio especialmente importante para la obtención de la acreditación de alta calidad de los programas. También quedará para un estudio posterior determinar si la composición de los programas con acreditación de alta calidad determina el desempeño de sus egresados.

\section{Implicaciones de la nueva legislación para las licenciaturas en Colombia}

En esta última sección señalaremos las implicaciones que tendrá en los programas de filosofía en Colombia la entrada en vigencia del Decreto 2450 de 2015 y la Resolución 2041 de 2016 del MEN, que reglamentan las condiciones de calidad de las licenciaturas en Colombia. Esta nueva reglamentación obedece a lo establecido en la Ley 1753 de 2015, "Plan nacional de desarrollo 2104-2018", según la cual todas las licenciaturas en Colombia deben obtener acreditación de alta calidad en un transcurso de dos años. Esta medida obedece en gran medida a los hallazgos encontrados en el estudio realizado por García et al. (2014) que evidencian que el bajo desempeño de la educación básica en 
Colombia obedece a una baja calidad en la formación de maestros.

Ahora bien, en lo que respecta a los programas de filosofía en Colombia, lo primero que hay que notar es que de las licenciaturas en filosofía solamente 7 de las 28 cuentan con acreditación de alta calidad en este momento. Esto implicará que, aunque todas se presenten al proceso de acreditación, no todas recibirán el registro de alta calidad, por lo que, de acuerdo con la normatividad, tendrán que cerrarse. Debido a que en las instituciones en las que se ofrecen algunas de estas licenciaturas que no cuentan con acreditación de alta calidad en la actualidad, ofrecen a su vez doble programa, junto con el profesional, en caso de que se llegasen a cerrar podría suceder que también se cerrara el programa profesional; aunque también es probable que solo se cierren algunas licenciaturas, pero se fortalezcan o aumenten los programas profesionales. Los programas tendrán dos años para aplicar la normatividad; por lo que en un lapso de tres años se recomienda llevar a cabo un estudio transversal para examinar con detalle los efectos sobre estos programas.

A nivel curricular más específico, el Decreto 2450 de 2015 exige de las licenciaturas el aumento de las estrategias de flexibilidad en las licenciaturas (MEN, art. 3.b), que en general es bajo, como lo muestran los bajos promedios de créditos en electivas libres o en profundización ( $c f$. Farieta, Gómez y Almeida, 2015). La norma también exige una implementación de estrategias de interdisciplinarización del programa (art. 3.1), lo que afectaría principalmente a las 15 licenciaturas que son únicamente en filosofía, pero no a las otras 13 que combinan filosofía con otras disciplinas ( $c f$. tabla 15), las cuales ya cuentan con un importante componente interdisciplinar. Sin embargo, estas licenciaturas que combinan filosofía con otras disciplinas se ven afectadas por el art. 2.1 de la Resolución 2041 de 2016, en la que se regulan los nombres para las licenciaturas y solo se aceptan, en el área de "Educación en ética y valores humanos" a la Licenciatura en Filosofía, la Licenciatura en Filosofía y Letras, y la Licenciatura en Ética y
Valores. La resolución obligaría entonces a 9 programas a cambiar su denominación.

El cambio más importante, a nivel de plan de estudios tendrá que ver con la necesidad de responder al requerimiento de cincuenta (50) créditos en prácticas para todas las licenciaturas (Resolución 2041 de 2016, art. 3.3). En la actualidad ningún programa cumple con este requisito y todos están muy lejos; el máximo de créditos es 15 (código SINES 13218) y la media para todas las licenciaturas es de apenas 7,75 créditos (cf. tabla 5). Esto implicará que todos los programas tendrán que hacer reformas sustantivas de sus planes de estudio y posiblemente tendrán que aumentar la duración y el número de créditos de los programas. Tal vez pasemos a tener licenciaturas de 10 semestres y con un mínimo de 160 créditos. En la actualidad solamente las licenciaturas de universidades públicas cumplen con estas condiciones (cf. Farieta, Gómez y Almeida, 2015), por lo que las privadas serán las que más extiendan su duración.

Los otros programas que se verán fuertemente afectados en su conformación curricular serán las 7 licenciaturas a distancia. De acuerdo con la Resolución 2041 de 2016 (MEN, art. 3.3), todas las licenciaturas deberán ofrecer al menos 40 créditos presenciales, y para universidades con acreditación de alta calidad, al menos 20 créditos. Esto quiere decir que las licenciaturas ciento por ciento virtuales tendrán que cumplir con este requisito y pasar a ser semipresenciales, o serán cerradas. Dado que el requerimiento es más bajo para las universidades con acreditación de alta calidad, es más probable que sean estas instituciones quienes sostengan sus programas, mientras que las demás, o pasen a la modalidad presencial o desaparezcan definitivamente.

Quedan muchos interrogantes con respecto al futuro de la educación profesional en filosofía en Colombia. Estas nuevas reglamentaciones pueden no solo afectar a las licenciaturas, sino que también a los programas presenciales. También, que universidades que ya cuentan con acreditación de alta calidad abran nuevas licenciaturas en filosofía, o con énfasis en filosofía; como acaba de hacer la Universidad de los Andes, la cual en julio de 2016 
abrió seis nuevas licenciaturas, entre ellas una en Ciencias Sociales con profundización en Filosofía, que no fue incluida en el presente estudio por su creación tan reciente. Serán necesarios varios estudios transversales en los próximos 10 a 15 años para poder evaluar en definitiva si estas nuevas políticas públicas en educación realmente han mejorado las condiciones de la enseñanza, tanto en la educación básica y media en general como en filosofía y sus áreas y disciplinas cercanas en Colombia. Hemos presentado solamente un punto de partida para la evaluación, pero esperamos en unos años poder hacer un seguimiento y dar veredictos con criterios objetivos sobre la eficacia de la política pública o, en el peor de los casos, la necesidad de que esta nuevamente sea replanteada.

\section{Referencias}

Colegio Mayor de Nuestra Señora del Rosario (s.f.) Filosofía. Plan de estudios. Recuperado de http:// www.urosario.edu.co/Filosofia/el-programa/ plan-de-estudios/\#verticalTab2.

Colombia. Congreso de la República. Ley 115 de 1994. Por la cual se expide la Ley General de Educación. Diario Oficial 41214. Recuperado de http://www.secretariasenado.gov.co/senado/basedoc/ley_0115_1994.html.

Congreso de la República (28 de diciembre de 1992). Ley 30. Por la cual se organiza el servicio público de la educación superior. Diario Oficial 40700. Recuperado de http://www.alcaldiabogota.gov.co/ sisjur/normas/Norma1.jsp?i=253.

Congreso de la República (9 de junio de 2015). Ley 1753. Por la cual se expide el Plan Nacional de Desarrollo 2014-2018 “Todos por un nuevo país”. Diario Oficial 49538. Recuperado de http://www.secretariasenado. gov.co/senado/basedoc/ley_1753_2015.html

Consejo Nacional de Acreditación (2013). Lineamientos para la acreditación de programas de pregrado. Bogotá: Ministerio de Educación Nacional. Recuperado de http://www.cna.gov.co/1741/articles-186359_pregrado_2013.pdf.

Educación Superior. Diario Oficial 40700. Recuperado de http://www.alcaldiabogota.gov.co/sisjur/normas/ Norma1.jsp?i=253.

Colombia. Congreso de la República (1994 febrero 8). Ley 115. Por la cual se expide la Ley General de
Educación. Diario Oficial 41214. Recuperado de http://www.secretariasenado.gov.co/senado/basedoc/ley_0115_1994.html.

Colombia. Congreso de la República (2015, junio 9). Recuperado de http://www.secretariasenado.gov.co/ senado/basedoc/ley_1753_2015.html.

Colombia. Ministerio de Educación Nacional (2010 abril 20). Decreto 1295Recuperado de http://www. alcaldiabogota.gov.co/sisjur/normas/Norma 1 . $j s p ? i=39363$.

Colombia. Ministerio de Educación Nacional (2015 mayo 26).Recuperado de http://www.alcaldiabogota.gov. co/sisjur/normas/Norma1.jsp?i=62510\#0

Colombia. Ministerio de Educación Nacional (2015 diciembre 17).Recuperado de http://www.alcaldiabogota.gov.co/sisjur/normas/Norma1.jsp? $\mathrm{i}=64184$.

Colombia. Ministerio de Educación Nacional (2016 febrero 3).Recuperado de http://www.mineducacion. gov.co/1759/articles-356144_recurso_1.pdf.

Corporación Universitaria Minuto deDios(s.f.). Estudiosen Filosofía. Plan de estudios. Recuperado de http://www. uniminuto.edu/documents/935233/1639769/2049. pdf/d7bd4597-4fca-484f-82a3-6fce7160c147.

Corporación Universitaria Minuto de Dios (s.f.). Licenciatura en Filosofía. Plan de estudios. Recuperado de http://www.uniminuto.edu/documents/ 935233/1639769/9363.pdf/e350c988-1cf9-4c67b43c-8ae73d25cd73.

Escanés, G., Herrero, V., Merlino, A. y Ayllón, S. (2014). Deserción en educación a distancia: factores asociados a la elección de modalidad como desencadenantes del abandono universitario. Virtualidad, Educación y Ciencia, 9(5), 45-55. Recuperado de https://revistas.unc.edu.ar/index.php/vesc/article/ view/9549/10318.

Escobar, J. (2010). El griego y el latín en la conformación del pensar como ciencia. Universitas Philosophica, 27(55), 233-253. Recuperado de http://www.scielo.org.co/scielo.php?script=sci_ arttext\&pid=S0120-53232010000200013\&lng=en $\& \operatorname{lng}=\mathrm{es}$.

Farieta, A., Gómez, M. y Almeida, S. (2015). Análisis comparativo de los planes de estudio de filosofía en Colombia. Parte 1: generalidades y flexibilidad. Folios, 42, 87-104. Recuperado de http:// revistas.pedagogica.edu.co/index.php/RF/article/ view/3162/2797 
Fundación Universitaria Católica del Norte (s.f.). Licenciatura en Filosofía y Educación Religiosa. Plan de estudios. Recuperado de: http://www.ucn.edu.co/ programas-academicos/Documents/Pensum2016/ LicenciaturaFilosofiaReligiosa.pdf.

Fundación Universitaria Católica Lumen Gentium. (s.f.). Licenciatura en Filosofía y Ciencias Religiosas. Plan de estudios. Recuperado de http://www.unicatolica. edu.co/web/site/rutas/FCR.pdf.

Fundación Universitaria Luis Amigó. (s.f.). Filosofía. Plan de estudios. Recuperado de http://www.funlam.edu. co/uploads/programas/pregrados/Filosofia-MED.pdf.

Fundación Universitaria Luis Amigó. (s.f.) Licenciatura en Filosofía. Plan de estudios. Recuperado de http:// www.funlam.edu.co/uploads/programas/pregrados/ LicenciaturaFilosofia-MED.pdf.

Fundación Universitaria San Alfonso (s.f.). Licenciatura en Filosofía. Plan de estudios. Recuperado de: http:// www.fusa.edu.co/archivos/programa_licenciatura_filosofia.pdf.

Fundación Universitaria San Alfonso. (2016) Filosofía Teología. Plan de estudios. Recuperado de http://www.sanalfonso.edu.co/\#!pregrado-licenciatura-en-filosofia/ cue8.

García, S., Maldonado, D., Perry, G., Rodríguez, C. y Saavedra, J.E. (2014) Tras la excelencia docente. Cómo mejorar la calidad de la educación para todos los colombianos. Bogotá: Fundación Compartir; Punto Aparte. Recuperado de http://compartirpalabramaestra.org/documentos/tras_la_excelencia_docente.pdf

Gutiérrez, M. y Flórez, R. (2012). Enseñar a escribir en la universidad: saberes y prácticas de docentes y estudiantes universitarios. Magis. Revista Internacional de Investigación en Educación, 4(7), 137-168. Recuperado de http://revistas.javeriana. edu.co/index.php/MAGIS/article/view/3561/2675.

Jámblico (2003). Vida pitagórica. Trad. M. Periago L. Madrid: Gredos.

Mann, H.B. y Whitney, D.R. (1947). On a Test of Whether one of Two Random Variables is Stochastically Larger than the Other. Annals of Mathematical Statistics, 18(1), 50-60.

Ministerio de Educación Nacional (3 de febrero de 2016). Resolución 2041. Por la cual se establecen las características específicas de calidad de los programas de Licenciatura para la obtención, renovación o modificación del registro calificado. Recuperado de: http:// www.mineducacion.gov.co/1759/articles-356144_recurso_1.pdf.

Ministerio de Educación Nacional (MEN) (17 de diciembre de 2015). Decreto 2450. Por el cual se reglamentan las condiciones de calidad para el otorgamiento y renovación del registro calificado de los programas académicos de licenciatura y los enfocados a la educación, y se adiciona el Decreto 1075 de 2015, Único Reglamentario del Sector Educación. Diario Oficial 49729. Recuperado de http://www.alcaldiabogota. gov.co/sisjur/normas/Norma1.jsp? $\mathrm{i}=64184$.

Ministerio de Educación Nacional (MEN) (20 de abril de 2010). Decreto 1295. Por el cual se reglamenta el registro calificado de que trata la Ley 1188 de 2008 y la oferta y desarrollo de programas académicos de educación superior. Diario Oficial 47.687. Recuperado de http://www.alcaldiabogota.gov.co/sisjur/normas/ Norma1.jsp?i=39363.

Ministerio de Educación Nacional (MEN) (26 de mayo de 2015). Decreto 1075. Por medio del cual se expide el Decreto Único Reglamentario del Sector Educación. Recuperado de http://www.alcaldiabogota.gov.co/ sisjur/normas/Norma1.jsp? $\mathrm{i}=62510 \# 0$

Morel, T. (2004) La interacción en la clase magistral. Alicante: Universidad de Alicante.

Navarro, N. (1983). El seminario investigativo. Bogotá: Ascún.

Navarro, F. (2013). Trayectorias de formación en lectura y escritura disciplinar en carreras universitarias de humanidades: diagnóstico y propuesta institucional. Revista Mexicana de Investigación Educativa, 18(58), 709-734. Recuperado de http://www.scielo.org.mx/scielo.php?script=sci_ arttext\&pid=S1405-66662013000300003\&lng=es\& tlng=en.

Ordóñez, A. (2005). Anotaciones sobre el seminario investigativo. Bogotá: PUJ.

Pablo VI (20 de octubre de 1965). Decreto Optatam Totius. Sobre la formación sacerdotal. Vaticano. Recuperado de http://www.vatican.va/archive/ hist_councils/ii_vatican_council/documents/ vat-ii_decree_19651028_optatam-totius_sp.html.

Pérez, R., Galán, A. y Quintanal, J. (2012). Métodos y diseños de investigación en educación. Madrid: UNED.

Pontificia Universidad Javeriana - Sede Cali. (s.f.) Licenciatura en Filosofía. Plan de estudios. Recuperado de http://www.javerianacali.edu.co/sites/ujc/files/ plan_de_estudios_carrera_de_filosofia.pdf. 
Pontificia Universidad Javeriana. (s.f.) Filosofía. Plan de estudios. Recuperado de http://puj-portal.javeriana.edu.co/portal/page/portal/Facultad $\% 20 \mathrm{de} \% 20$ Filosof\%EDa/plt_lic_filosofia/Plan\%20de $\% 20$ estudios.

Pontificia Universidad Javeriana. (s.f.) Licenciatura en Filosofía. Plan de estudios. Recuperado de http://pujportal.javeriana.edu.co/portal/page/portal/Facultad $\% 20$ de\%20Filosof\%EDa/plt_carrera_filosofia/Plan $\% 20$ de\%20estudios.

Qu, X. (2015) Comparison of undergraduate geology courses in China and recommendations for change. World Transactions on Engineering and Technology Education, 13(4), pp. 609-614. Recuperado de https:// www.scopus.com/inward/record.uri?eid=2-s2.0$84953715928 \&$ partnerID $=40 \& \mathrm{md} 5=8 \mathrm{eb} 5830 \mathrm{~d} 30 \mathrm{a}$ 0a9a14e6d6fac8be46c48

Ramírez, M.A. y Casillas, M. (coords.) (2014). Háblame de TIC. Tecnología digital en la Educación Superior. Argentina: Brujas. Recuperado de http://www.uv.mx/ personal/albramirez/files/2014/08/hablamedeTIC_ librocompleto.pdf.

Rodríguez S., M. (2011) Metodologías docentes en el EEES: de la clase magistral al portafolio. Tendencias Pedagógicas, 17, 83-103. Recuperado de https:// revistas.uam.es/tendenciaspedagogicas/article/ view/1961/2072.

Schalk, A.E. (2010). El impacto de las TIC en la educación. Relatoría de la Conferencia Internacional de Brasilia, 26-29 abril de 2010. Santiago de Chile: Unesco. Recuperado de http://unesdoc.unesco.org/ images/0019/001905/190555s.pdf.

Seel, N.M. (2012). Epistemology and Learning in Medieval Philosophy. En N.M. Seel (ed.), Encyclopedia of the Sciences of Learning (pp. 1168-1171). Boston, MA: Springer.

Unesco Institute for Statistics (2009). Medición de las tecnologías de la información y la comunicación (TIC) en educación: manual del usuario. París: Unesco. Recuperado de http://unesdoc.unesco.org/ images/0018/001883/188309s.pdf.

Universidad Católica de Oriente (2013). Licenciatura en Filosofía y Educación Religiosa. Recuperado de http://www.uco.edu.co/educacion/filosofia/Paginas/ proyecto-educativo-institucional.aspx

Universidad de Antioquia (s.f.) Licenciatura en Filosofía. Plan de estudios. Recuperado de http://www.udea.
edu.co/portal/page/portal/SedesDependencias/ Filosofia/C.estudiarInstituto/A.ProgramasPregrado.

Universidad de Antioquia. (s.f.) Filosofía. Plan de estudios. Recuperado de http://www.udea.edu.co/ portal/page/portal/SedesDependencias/Filosofia/C. estudiarInstituto/A.ProgramasPregrado.

Universidad de Caldas. (s.f.) Filosofía y Letras. Plan de estudios. Recuperado de http://acad.ucaldas.edu. co/gestionacademica/planestudios/pensumver. asp? cod=412\&cod_carrera $=025$.

Universidad de Caldas. (s.f.) Licenciatura en Filosofía y Letras. Plan de estudios. Recuperado de http://acad. ucaldas.edu.co/gestionacademica/planestudios/pensumver.asp?cod_carrera $=213$.

Universidad de Cartagena. (s.f.) Filosofía. Proyecto Educativo del Programa. Recuperado de http:// filosofia.unicartagena.edu.co/index.php/inicio/elprograma/item/306-proyecto-educativo-del-programa-de-filosofia

Universidad de la Sabana. (2010) Filosofía. Plan de estudios. Recuperado de: http://www.unisabana. edu.co/fileadmin/Documentos/Filosofia/plan_estudios_2010.pdf.

Universidad de la Salle. (s.f.) Filosofía y Letras. Plan de estudios. Recuperado de http://unisalle.lasalle.edu. co/media/docs/programas-academicos/pregrado/ malla-curricular-filosofia-y-letras.pdf.

Universidad de los Andes. (s.f.) Filosofía. Plan de estudios. Recuperado de http://filosofia.uniandes.edu.co/ index.php/programas/pregrado/descripcion.

Universidad de Nariño. (s.f.) Licenciatura en Filosofía y Letras. Plan de estudios. Recuperado de http://pregrado.udenar.edu.co/? $\mathrm{p}=144$.

Universidad de Pamplona. (2013) Filosofía. Plan de estudios. Recuperado de http://www.unipamplona.edu. co/unipamplona/portalIG/home_1/recursos/facultades/artes/13092013/pemsun_nuevo_filosofia.xls.

Universidad de San Buenaventura. (s.f.) Licenciatura en Filosofía. Plan de estudios. Recuperado de http:// www.usbbog.edu.co/index.php/programas-academicos/carreras-profesionales/facultad-de-filosofia/ licenciatura-en-filosofia/plan-de-estudios.

Universidad del Atlántico. (s.f.) Filosofía. Plan de estudios. Recuperado de http://www.uniatlantico.edu.co/ uatlantico/docencia/ciencias-humanas/programas/ filosofia. 
Universidad del Cauca. (2012) Filosofía. Plan de estudios. Recuperado de http://www.unicauca.edu.co/ versionP/oferta-academica/programas-de-pregrado/ filosofia/plan-de-estudios.

Universidad del Norte. (2015). Filosofía y Humanidades. Plan de estudios. Recuperado de http://www. uninorte.edu.co/web/filosofiayhumanidades/ plan-de-estudios.

Universidad del Norte. (2016). Licenciatura en Filosofía y Humanidades. Plan de estudios. Recuperado de http: //www.uninorte.edu.co/web/licenciatura-enfilosofia-y-humanidades/plan-de-estudio.

Universidad del Quindío. (2014) Filosofía. Plan de estudios. Recuperado de https://www.uniquindio.edu. co/filosofia/publicaciones/plan_de_estudio_17_pub.

Universidad del Valle. (2013) Licenciatura en Filosofía. Plan de estudios. Recuperado de http://paginasweb. univalle.edu.co/ filosofia/doc/profesional/PEP_ PROFESIONAL.pdf

Universidad del Valle. (2015) Profesional en Filosofía. Estructura curricular. Recuperado de http://paginasweb.univalle.edu.co/ filosofia/Profesional_ Filosofia/estructura_curricular.html.

Universidad El Bosque. (s.f.) Filosofía. Plan de estudios. Recuperado de http://www.uelbosque.edu.co/ programas_academicos/pregrado/filosofia.

Universidad Externado de Colombia. (s.f.) Filosofía. Plan de estudios. Recuperado de http://190.7.110.123/ pdf/1_facultadCienciasSocialesHumanas/2012/10/ filosofia.pdf.

Universidad Industrial de Santander. (s.f.) Filosofía. Plan de estudios. Recuperado de http://www.uis.edu.co/ webUIS/es/academia/facultades/cienciasHumanas/ escuelas/filosofia/programasAcademicos/filosofia/ planEstudios.html.

Universidad La Gran Colombia. (s.f.) Licenciatura en Filosofía e Historia. Plan de estudios. Recuperado dehttp://www.ugc.edu.co/documentos/admisiones/ mallas/Malla_Filosofia_e_Historia.pdf.

Universidad Libre. (s.f.) Filosofía. Plan de estudios. Recuperado de http://www.unilibre.edu.co/bogota/ ul/noticias/noticias-universitarias/1820-pregradofilosofia\# estructura-curricular.

Universidad Nacional Abierta y a Distancia. (s.f.) Filosofía. Plan de estudios. Recuperado de http:// estudios.unad.edu.co/images/ecsah/Filosofia/ Filosofia_-_Mapa_curricular_Plan_2014.ppt.
Universidad Nacional Abierta y a Distancia. (s.f.) Licenciatura en Filosofía. Plan de estudios. Recuperado de https://estudios.unad.edu.co/images/ecedu/archivosEscuela/Plan_de_estudios.pdf.

Universidad Nacional de Colombia. (s.f.) Filosofía. Plan de estudios. Recuperado de http://www.humanas.unal.edu.co/filosofia/programas-curriculares/ pregrado/.

Universidad Pedagógica Nacional. (2014) Licenciatura en Filosofía. Plan de estudios. Recuperado de http:// www.pedagogica.edu.co/admin/UserFiles/malla_curricular_licenciatura_filosofia(1).pdf .

Universidad Pedagógica y Tecnológica de Colombia. (s.f.) Licenciatura en Filosofía. Plan de estudios. Recuperado de http://www.uptc.edu.co/facultades/f_educacion/ pregrado/filosofia/inf_general/.

Universidad Pontificia Bolivariana. (s.f.) Licenciatura en Filosofía y Letras. Plan de estudios. Recuperado de http://www.upb.edu.co/portal/page?_ pageid $=1054,32439378 \&$ dad $=$ portal\&_schema $=$ PORTAL.

Universidad Pontificia Bolivariana. (2012) Filosofía. Proyecto Educativo de Programa. Recuperado de http:// www.upb.edu.co/pls/portal/docs/PAGE/GPV2_UPB_ MEDELLIN/PGV2_M030_PREGRADOS/PGV2_ M030080040_FILOSOF\%CDA/PROYECTO $\% 20$ EDUCATIVO\%20DEL\%20PROGRAMA\%20 -PEP-\%20FILOSOFIA.PDF.

Universidad Pontificia Bolivariana. (s.f.) Filosofía y Letras. Plan de estudios. Recuperado de http://www.upb. edu.co/portal/page?_pageid $=1054,32439378 \&$ _ dad=portal\&_schema=PORTAL.

Universidad Santo Tomas. (s.f.) Licenciatura en Filosofía y Educación Religiosa. Plan de estudios. Recuperado de http://www.ustadistancia.edu.co/images/stories/PLAN_DE_ESTUDIOS_FIL_EDUCACION_ RELIGIOSA.pdf

Universidad Santo Tomas. (s.f.) Licenciatura en Filosofía. Ética y Valores Humanos. Plan de estudios. Recuperado de http://www.ustadistancia.edu. co/images/stories/PLAN_DE_ESTUDIOS_FIL_ ETICA_Y_VALORES.pdf

Universidad Santo Tomas. (s.f.) Licenciatura en Filosofía y Lengua Castellana. Plan de estudios. Recuperado de http://filosofia.usta.edu.co/images/stories/dependencia/pregrado/documentos/ pensum_v2.pdf. 
Universidad Santo Tomas (s.f.). Licenciatura en Filosofía. Pensamiento Político y Económico. Plan de estudios. Recuperado de http://www.ustadistancia.edu. co/images/stories/PLAN_DE_ESTUDIOS_FIL_ PENSAMIENTO_POLITICO_Y_ECONOMICO.pdf.

Universidad Sergio Arboleda. (2011) Licenciatura en Filosofía y Humanidades. Plan de estudios. Recuperado de http://www.usergioarboleda.edu.co/wp-content/ uploads/2015/04/Licenciatura-Filosofia.pdf.

Universidad Sergio Arboleda. (2015) Filosofía y Humanidades. Plan de estudios. Recuperado de http://www.usergioarboleda.edu.co/wp-content/ uploads/2015/04/Filosofia11.pdf.

Universidad Tecnológica de Pereira. (2011) Licenciatura en Filosofía. Plan de estudios. Recuperado de http://media.utp.edu.co/planeacion/archivos/documentos-de-interes-de-planeacion-academica/ documentocondicionesiniciales-utp-julio-2009final1.pdf.

Universitaria Agustiniana. (2012) Licenciatura en Filosofía. Plan de estudios. Recuperado de http://www.uniagustiniana.edu.co/index.php/plan-de-estudios-licenciatura-en-filosofia.html.

Vanegas, N., Valencia, T.; Reinoso, J. y Cortés, M. (2013). Utilización de las TIC en los programas actuales $y$ nuevos programas. Documento de trabajo. Cali: Pontificia Universidad Javeriana. Recuperado de http://www.javerianacali.edu.co/sites/ujc/files/node/ field-documents/field_document_file/utilizacion_ de_las_tic_en_los_programas_actuales_y_nuevos_ programas.pdf.

Wilcoxon, F. (1945). Individual comparisons by Ranking Methods. Biometrics Bulletin, 1, 80-83. Recuperado de: http://www.jstor.org/stable/3001968. 